\title{
Random attractors for the stochastic coupled suspension bridge equations of Kirchhoff type
}

Ling $\mathrm{Xu}^{{ }^{1 *} \text {, Jianhua Huang }}{ }^{1}$ and Qiaozhen $\mathrm{Ma}^{2}$

"Correspondence:

13893414055@163.com

${ }^{1}$ College of Liberal Arts and

Sciences, National University of Defense Technology, Changsha,

P.R. China

Full list of author information is

available at the end of the article

\begin{abstract}
This paper is devoted to the dynamical behavior of stochastic coupled suspension bridge equations of Kirchhoff type. For the deterministic cases, there are many classical results such as existence and uniqueness of a solution and long-term behavior of solutions. To the best of our knowledge, the existence of random attractors for the stochastic coupled suspension bridge equations of Kirchhoff type is not yet considered. We intend to investigate these problems. We first obtain the dissipativeness of a solution in higher-energy spaces

$H^{3}(U) \times H_{0}^{1}(U) \times\left(H^{2}(U) \cap H_{0}^{1}(U)\right) \times H_{0}^{1}(U)$. This implies that the random dynamical system generated by the equation has a random attractor in $\left(H^{2}(U) \cap H_{0}^{1}(U)\right) \times L^{2}(U) \times H_{0}^{1}(U) \times L^{2}(U)$, which is a tempered random set in the space in $H^{3}(U) \times H_{0}^{1}(U) \times\left(H^{2}(U) \cap H_{0}^{1}(U)\right) \times H_{0}^{1}(U)$.
\end{abstract}

Keywords: Coupled suspension bridge equations; Random dynamical system; Random attractors; Kirchhoff-type

\section{Introduction}

In this paper, for simplicity, set $\Delta^{2} u=u_{x x x x},-\Delta u=-u_{x x}, \nabla u=u_{x}$. We consider the following stochastic coupled suspension bridge equations of Kirchhoff type:

$$
\left\{\begin{array}{l}
u_{1 t t}+\alpha \triangle^{2} u_{1}+\delta_{1} u_{1 t}+k\left(u_{1}-u_{2}\right)^{+}+\left(p-\left\|\nabla u_{1}\right\|_{L^{2}(U)}^{2}\right) \Delta u_{1}+f_{B}\left(u_{1}\right)=q_{B}(x) \dot{W}_{1}, \\
\quad \text { in } U \times[\tau,+\infty), \tau \in \mathbb{R}, \\
u_{2 t t}-\beta \triangle u_{2}+\delta_{2} u_{2 t}-k\left(u_{1}-u_{2}\right)^{+}+f_{S}\left(u_{2}\right)=q_{S}(x) \dot{W}_{2}, \quad \text { in } U \times[\tau,+\infty), \tau \in \mathbb{R}
\end{array}\right.
$$

subject to boundary conditions at both ends

$$
\begin{aligned}
& u_{1}(x, t)=\Delta u_{1}(x, t)=0, \quad x \in \partial U, t \geq \tau, \\
& u_{2}(x, t)=0, \quad x \in \partial U, t \geq \tau,
\end{aligned}
$$

and the initial-value conditions

$$
\begin{array}{ll}
u_{1}(x, \tau)=u_{10}(x), & u_{1 t}(x, \tau)=u_{11}(x), \quad x \in U, \\
u_{2}(x, \tau)=u_{20}(x), & u_{2 t}(x, \tau)=u_{21}(x), \quad x \in U,
\end{array}
$$

(c) The Author(s) 2019. This article is distributed under the terms of the Creative Commons Attribution 4.0 International License (http://creativecommons.org/licenses/by/4.0/), which permits unrestricted use, distribution, and reproduction in any medium, provided you give appropriate credit to the original author(s) and the source, provide a link to the Creative Commons license, and indicate if changes were made. 
Figure 1 A model of the one-dimensional bridge represented by the coupling of the cable (a vibrating string) and the roadbed (a vibrating beam) by the stays, treated as nonlinear springs

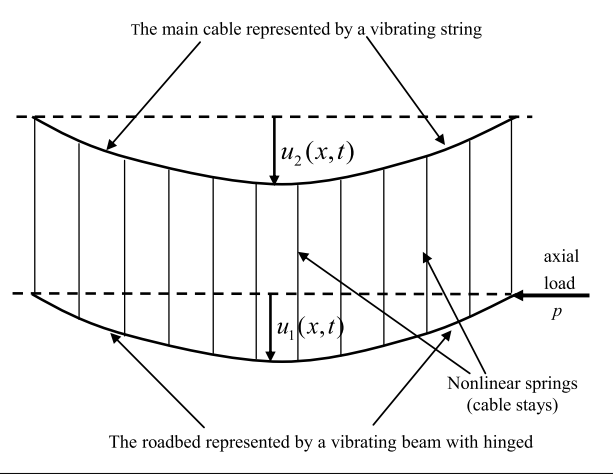

where $U$ is a bounded closed interval of $R$ with a boundary $\partial U=\{0, L\}$. The first equation of (1.1) represents the vibration of the road bed in the vertical direction, and the second equation describes that of the main cable from which the road bed is suspended by the tie cables, see $[1,2]$. We assume the ratios between the length of the bridge and other dimensions to be very small, which entails that the torsional motion can be ignored, so that the road bed can be simply modeled as a vibrating one-dimensional beam. In addition, by neglecting the influence of the towers and side parts of the bridge, such a beam can be assumed to have simply supported ends (see Fig. 1).

Now, we introduce the meanings of each terms in (1.1) as follows.

- $u_{1}=u_{1}(x, t):[0, L] \times[\tau,+\infty) \rightarrow \mathbb{R}$ represents the downward deflection of the beam mid-line (of unitary length) in the vertical plane with respect to the reference configuration.

- $u_{2}=u_{2}(x, t):[0, L] \times[\tau,+\infty) \rightarrow \mathbb{R}$ measures the vertical displacement of the string.

$-\alpha>0$ and $\beta>0$ are the flexural rigidity of the structure and coefficient of tensible strength of the cable, respectively.

- $\delta_{1}, \delta_{2}>0$ represent the viscous damping constants due to external resistant forces which linearly depend on the velocity.

$-k>0$ is the spring constant, the nonlinear terms $\pm k\left(u_{1}-u_{2}\right)^{+}$model the restoring force due to the suspenders, which are assumed to behave as one-sided springs. Such a restoring force is proportional to the elongation of the suspenders if they are stretched and vanishes if they are compressed. In addition, it holds the road bed up and pulls the cable down; therefore, into the first equation, the plus sign in the front of $k\left(u_{1}-u_{2}\right)^{+}$occurs, but into the second equation, the sign in front of the same term is minus.

- $p \in \mathbb{R}$ accounts for the axial force acting at the end of the road bed of the bridge in the reference configuration: it is negative when the beam is axially stretched, positive when compressed.

- $\left\|\nabla u_{1}\right\|_{L^{2}(U)}^{2}$ takes into account the geometry of the beam bending due to its elongation.

- $f_{B}$ and $f_{S}$ are given vertical dead-load distributions acting on the deck and the main cable, respectively.

In addition to normal vehicular load, a bridge is also occasionally subject to random loads such as seismic and wind forces. Such random forces can be modeled by two noise terms $\dot{W}_{1}(t)$ and $\dot{W}_{2}(t)$ on the right-hand side of equation $(1.1)$. $\dot{W}_{i}(t)(i=1,2)$ are the scalar Gaussian white noises, i.e., formally the derivative of the two-sided real-value scalar 
Wiener processes $\left\{W_{i}(t)\right\}_{t \in \mathbb{R}}(i=1,2)$. We assume that the functions $f_{B}, f_{S}, q_{B}, q_{S}$ always satisfy the following assumptions.

(i) The nonlinear terms $f_{B} \in C^{3}(\mathbb{R}, \mathbb{R})$ and $f_{S} \in C^{2}(\mathbb{R}, \mathbb{R})$ with $f_{B}(0)=f_{S}(0)=0$, which satisfy the following conditions.

(a) Growth conditions:

$$
\left|f_{B}(\tau)\right|,\left|f_{S}(\tau)\right| \leq C_{0}\left(1+|\tau|^{\gamma}\right), \quad \gamma \geq 1, \forall \tau \in \mathbb{R}
$$

where $C_{0}$ is a positive constant.

(b) Dissipation conditions:

$$
\begin{aligned}
& F_{B}(\tau):=\int_{0}^{\tau} f_{B}(r) d r \geq C_{1}\left(|\tau|^{\gamma+1}-1\right), \\
& F_{S}(\tau):=\int_{0}^{\tau} f_{S}(r) d r \geq C_{1}\left(|\tau|^{\gamma+1}-1\right), \quad \gamma \geq 1, \forall \tau \in \mathbb{R},
\end{aligned}
$$

and

$$
\tau f_{B}(\tau) \geq C_{2}\left(F_{B}(\tau)-1\right), \quad \tau f_{S}(\tau) \geq C_{2}\left(F_{S}(\tau)-1\right), \quad \forall \tau \in \mathbb{R}
$$

where $C_{1}$ and $C_{2}$ are positive constants.

(ii) $q_{B}(x) \in H^{3}(U) \cap H_{0}^{1}(U)$ and $q_{S}(x) \in H^{2}(U) \cap H_{0}^{1}(U)$ are not identically equal to zero.

The suspension bridge equations were presented by Lazer and McKenna as new problems in the field of nonlinear analysis [1]. Equation (1.1) models a random perturbation of the coupled suspension bridge equation of Kirchhoff type. If we ignore the effects of white noises in (1.1), that is, $q_{B}(x) \equiv q_{S}(x) \equiv 0$, then there are a lot of profound results on the dynamics of a variety of deterministic systems related to (1.1), see [3-19] and the reference therein. For example, just for a single deterministic suspension bridge equation (without white noises), in $[3-9,18]$, the authors investigated the existence, uniqueness, and global attractors of the solution. And for the deterministic coupled string-beam equations (without white noises) and the similar problems, Woinowsky-Krieger gave existence and uniqueness of the solution in the different spaces [17]. The authors achieved the existence of strong solutions and global attractors for both the autonomous case in [13] and the nonautonomous case in [14]. Other results have been obtained in [10-12, 15, 16, 19] and the reference therein.

From the above presentation we can see that the universal attractors for the deterministic suspension bridge equations are better investigated. On the other hand, the random dynamics of the suspension bridge equations are little considered. Ahmed [20] presented the stochastic versions of the models used in [2] and also their extensions to models that include torsional motions. This permits to study suspension bridges subject to random wind or seismic forces in addition to deterministic loads. They proved the existence, uniqueness, and regularity properties of solutions of these stochastic models on these Hilbert spaces. In case of sustained stochastic wind forces, it is conceivable that a random nonempty set as an attractor may exist and may lead to collapse of the bridge unless the attractor contains the rest state and the bridge returns to the rest state after the storm has passed. Therefore, it is necessary and meaningful to study the random attractors for 
the suspension bridge equation. Recently, the authors studied the existence of random attractors for both the single extensible suspension bridge equation in [21] and the coupled beam-string system in [22]. To the best of our knowledge, the existence of random attractors for the coupled suspension bridge equations of Kirchhoff type with white noises is still not considered, while it is just our concern.

The concept of random attractors was introduced in [23-25] for the infinite-dimensional random dynamical systems (RDS). A random attractor of RDS is a measurable and compact invariant random set attracting all orbits. It is the appropriate generalization of the now classical attractor from the deterministic dynamical systems to the RDS. The reason is that if such a random attractor exists, it is the smallest attracting compact set and the largest invariant set [25]. These abstract results have been successfully applied into many stochastic dissipative partial differential equations such as reaction diffusion equations, Navier-Stokes equations, and nonlinear wave equations, see [26-30] and the references therein. For instance, the existence of random attractor for a damped sine-Gordon equation was proved in [26]. Yang, Kloeden, and Duan [28, 29] studied random attractors for the stochastic semi-linear degenerate parabolic equation and the wave equation with nonlinear damping and white noise, respectively.

As we know, for a dynamical system perturbed by a white noise, there is no chance that bounded subsets of the state space remain invariant. White noise pushes the system out of every bounded set with probability one [23, 24]. It implies that the classical results of global attractors for the deterministic dynamical system are not suitable for RDS. Therefore, how to establish a compact random invariant set is the main task in studying these problems. Considering our system (1.1)-(1.3), the parameter $p \in \mathbb{R}$ and the term $-\|\nabla u\|_{L^{2}(U)}^{2} \Delta u$ also bring some difficulties, they make the calculus more complex than those in $[22,30]$. In order to prove the existence of random attractors for system (1.1)-(1.3), we use the methods established by Crauel, Flandoli, Arnold, and others in [23-25], which are still vital and useful until now.

The article is organized as follows. The definition of RDS and some abstract results are stated in Sect. 2. In Sect. 3, we present the existence and uniqueness of the solution corresponding to system (1.1)-(1.3), which determines an RDS. Finally, the existence of random attractors is shown in Sect. 4.

In this paper, the letters $C_{i}(i \in \mathbb{N})$ below are generic positive constants which do not depend on $\omega, \tau$, and $t$.

\section{Necessary notations and abstract results}

In this section, we recall some basic concepts related to the RDS and random attractors for an RDS in [23-25], which are important for getting our main results.

Let $\left(X,\|\cdot\|_{X}\right)$ be a separable Hilbert space with Borel $\sigma$-algebra $\mathcal{B}(X)$, and let $(\Omega, \mathcal{F}, P)$ be a probability space, where

$$
\Omega=\left\{\omega(t) \in C\left(\mathbb{R}, \mathbb{R}^{2}\right): \omega(0)=0\right\}
$$

is endowed with compact-open topology, $P$ is a Wiener measure, and $\mathcal{F}$ is the Pcompletion of Borel $\sigma$-algebra on $\Omega$. In addition, we write $W(t, \omega)=\left(W_{1}(t), W_{2}(t)\right)^{T}=$ $\omega(t), t \in \mathbb{R}, \omega \in \Omega$ and define

$$
\theta_{t} \omega(\cdot)=\omega(\cdot+t)-\omega(t), \quad t \in \mathbb{R}, \omega \in \Omega .
$$


Then $\theta_{t}: \Omega \rightarrow \Omega, t \in \mathbb{R}$ is a family of measure preserving and ergodic transformations such that $(t, \omega) \mapsto \theta_{t} \omega$ is measurable, $\theta_{0}=$ id and $\theta_{t+s}=\theta_{t} \theta_{s}$ for all $t, s \in \mathbb{R}$. The flow $\theta_{t}$ together with the probability space $\left(\Omega, \mathcal{F}, P,\left(\theta_{t}\right)_{t \in \mathbb{R}}\right)$ is called a metric dynamical system.

Definition 2.1 Let $\left(\Omega, \mathcal{F}, P,\left(\theta_{t}\right)_{t \in \mathbb{R}}\right)$ be a metric dynamical system. Suppose that the mapping $\phi: \mathbb{R}^{+} \times \Omega \times X \rightarrow X$ is $\left(\mathcal{B}\left(\mathbb{R}^{+}\right) \times \mathcal{F} \times \mathcal{B}(X), \mathcal{B}(X)\right)$-measurable and satisfies the following properties:

(i) $\phi(0, \omega) x=x, x \in X$, and $\omega \in \Omega$;

(ii) $\phi(t+s, \omega)=\phi\left(t, \theta_{s} \omega\right) \circ \phi(s, \omega)$ for all $t, s \in \mathbb{R}^{+}, x \in X$, and $\omega \in \Omega$.

Then $\phi$ is called a random dynamical system (RDS). Moreover, $\phi$ is called a continuous RDS if $\phi$ is continuous with respect to $x$ for $t \geq 0$ and $\omega \in \Omega$.

Definition 2.2 A set-valued map $D: \Omega \rightarrow 2^{X}$ is said to be a closed (compact) random set if $D(\omega)$ is closed (compact) for $P$-a.s. $\omega \in \Omega$, and $\omega \mapsto d(x, D(\omega))$ is $P$-a.s. measurable for all $x \in X$.

Definition 2.3 If $K$ and $B$ are random sets such that for $P$-a.s. $\omega$ there exists a time $t_{B}(\omega)$ such that, for all $t \geq t_{B}(\omega)$,

$$
\phi\left(t, \theta_{-t} \omega\right) B \subset K(\omega)
$$

then $K$ is said to absorb $B$, and $t_{B}(\omega)$ is called the absorption time.

Definition 2.4 A random set $\mathcal{A}=\{A(\omega)\}_{\omega \in \Omega} \subset X$ is called a random attractor associated with the RDS $\phi$ if $P$-a.s.:

(i) $\mathcal{A}$ is a random compact set, i.e., $A(\omega)$ is compact for $P$-a.s. $\omega \in \Omega$, and the map $\omega \mapsto \mathrm{d}(x, A(\omega))$ is measurable for every $x \in X$;

(ii) $\mathcal{A}$ is $\phi$-invariant, i.e., $\phi(t, \omega) A(\omega)=A\left(\theta_{t} \omega\right)$ for all $t \geq 0$ and $P$-a.s. $\omega \in \Omega$;

(iii) $\mathcal{A}$ attracts every bounded (non-random) set $B$ in $X$, i.e., for all bounded (and non-random) $B \subset X$,

$$
\lim _{t \rightarrow \infty} \mathrm{d}\left(\phi\left(t, \theta_{-t} \omega\right) B, A(\omega)\right)=0
$$

where $\mathrm{d}(\cdot, \cdot)$ denotes the Hausdorff semidistance:

$$
\mathrm{d}(A, B)=\sup _{x \in A} \inf _{y \in B} d(x, y), \quad A, B \in X .
$$

Note that $\phi\left(t, \theta_{-t} \omega\right) x$ can be interpreted as the position of the trajectory which was in $x$ at time $-t$. Thus, the attraction property holds from $t=-\infty$.

Theorem 2.5 ([14]; Existence of a random attractor) Let $\phi$ be a continuous random $d y$ namical system on $X$ over $\left(\Omega, \mathcal{F}, P,\left(\theta_{t}\right)_{t \in \mathbb{R}}\right)$. Suppose that there exists a random compact set $K(\omega)$ absorbing every bounded non-random set $B \subset X$. Then the set

$$
\mathcal{A}=\{A(\omega)\}_{\omega \in \Omega}=\overline{\bigcup_{B \subset X} \Lambda_{B}(\omega)}
$$


is a global random attractor for $\phi$, where the union is taken over all bounded $B \subset X$, and $\Lambda_{B}(\omega)$ is the $\omega$-limits set of $B$ given by

$$
\Lambda_{B}(\omega)=\bigcap_{s \geq 0} \overline{\bigcup_{t \geq s} \phi\left(t, \theta_{-t} \omega\right) B}
$$

\section{Existence and uniqueness of solutions}

Let $(H,(\cdot, \cdot),\|\cdot\|)$ be a real Hilbert space, and let $A: D(A) \rightarrow H$ be a strictly positive selfadjoint operator. For any $r \in \mathbb{R}$, the scale of Hilbert spaces generated by the powers of $A$ is introduced as follows:

$$
H^{r}=D\left(A^{\frac{r}{4}}\right), \quad(u, v)_{r}=\left(A^{\frac{r}{4}} u, A^{\frac{r}{4}} v\right),\|u\|_{r}=\left\|A^{\frac{r}{4}} u\right\| .
$$

When $r=0$, the index $r$ is omitted. In particular, we have the compact embeddings $H^{r+1} \hookrightarrow$ $H^{r}$ along with the generalized Poincré inequality, there holds

$$
\|u\|_{r+1}^{4} \geq \lambda_{1}\|u\|_{r}^{4}, \quad \forall u \in H^{r+1},
$$

where $\lambda_{1}>0$ is the first eigenvalue of $A$.

In order to establish more general results, we recast system (1.1)-(1.3) into an abstract setting. Without loss of generality, we define

$$
\begin{aligned}
& Y_{0}=L^{2}(U), \quad Y_{1}=H_{0}^{1}(U), \quad Y_{2}=H^{2}(U) \cap H_{0}^{1}(U), \\
& D(A)=\left\{u, \Delta u \in H^{2}(U) \mid u(0)=u(L)=\Delta u(0)=\Delta u(L)=0\right\},
\end{aligned}
$$

where $A=\Delta^{2}, A^{\frac{1}{2}}=-\Delta$. For brevity, we introduce some spaces $V_{1}, V_{2}, E$ which are used throughout the paper, that is,

$$
V_{1}=Y_{1} \times Y_{0}, \quad V_{2}=Y_{2} \times Y_{0}, \quad E=V_{2} \times V_{1},
$$

and endow spaces $V_{1}, V_{2}, E$ with the following norms, respectively:

$$
\begin{aligned}
& \left\|y_{1}\right\|_{V_{1}}^{2}=\left\|u_{1}\right\|_{2}^{2}+\left\|v_{1}\right\|^{2} ; \quad\left\|y_{2}\right\|_{V_{2}}^{2}=\left\|u_{2}\right\|_{1}^{2}+\left\|v_{2}\right\|^{2} ; \\
& \|y\|_{E}^{2}=\alpha\left\|u_{1}\right\|_{2}^{2}+\left\|v_{1}\right\|^{2}+\beta\left\|u_{2}\right\|_{1}^{2}+\left\|v_{2}\right\|^{2}
\end{aligned}
$$

for all $y_{i}=\left(u_{i}, v_{i}\right)^{T} \in V_{i}, y=\left(y_{1}, y_{2}\right)=\left(\left(u_{1}, v_{1}\right)^{T},\left(u_{2}, v_{2}\right)^{T}\right) \in E, i=1,2$, here $T$ denotes the transposition.

Let $u(x, t)=\left(u_{1}(x, t), u_{2}(x, t)\right)^{T}, \Phi=\left(\begin{array}{cc}\alpha A & O \\ O & \beta A^{\frac{1}{2}}\end{array}\right), Q(x)=\left(\begin{array}{cc}q_{B}(x) & 0 \\ 0 & q_{S}(x)\end{array}\right), \quad \Lambda=\left(\begin{array}{cc}\delta_{1} & 0 \\ 0 & \delta_{2}\end{array}\right), f(u)=$ $\left(f_{B}\left(u_{1}\right)+k\left(u_{1}-u_{2}\right)^{+}-\left(p-\left\|u_{1}\right\|_{1}^{2}\right) A^{\frac{1}{2}} u_{1}, f_{S}\left(u_{2}\right)-k\left(u_{1}-u_{2}\right)^{+}\right)^{T}, W(t)=\left(W_{1}(t), W_{2}(t)\right)^{T}$. Then system (1.1)-(1.3) can be written as

$$
\left\{\begin{array}{l}
u_{t t}+\Phi u+\Lambda u_{t}+f(u)=Q(x) \dot{W}(t), \quad \text { in } U \times[\tau,+\infty), \tau \in \mathbb{R}, \\
\left.u(x, t)\right|_{\partial U}=\left(\left.u_{1}(x, t)\right|_{\partial U},\left.u_{2}(x, t)\right|_{\partial U}\right)^{T}=(0,0)^{T}, \\
\left.\Delta u(x, t)\right|_{\partial U}=\left(\left.\Delta u_{1}(x, t)\right|_{\partial U}, 0\right)^{T}=0, \\
u(x, \tau)=u_{0}(x)=\left(u_{10}(x), u_{20}(x)\right)^{T}, \\
u_{t}(x, \tau)=u_{1}(x)=\left(u_{11}(x), u_{21}(x)\right)^{T} .
\end{array}\right.
$$


Let

$$
\varepsilon=\min \left\{\frac{\alpha \lambda_{1} \delta_{1}}{2 \delta_{1}^{2}+3 \alpha \lambda_{1}}, \frac{\beta \sqrt{\lambda_{1}} \delta_{2}}{2 \delta_{2}^{2}+3 \beta \sqrt{\lambda_{1}}}\right\}
$$

By the transformation, $u=u, z=\left(z_{1}, z_{2}\right)^{T}=\left(u_{1 t}+\varepsilon u_{1}-q_{B}(x) W_{1}(t), u_{2 t}+\varepsilon u_{2}-q_{S}(x) \times\right.$ $\left.W_{2}(t)\right)^{T}=u_{t}+\varepsilon u-Q(x) W(t)$, we obtain the following random partial differential equation (RPDE):

$$
\left\{\begin{array}{l}
\frac{d u}{d t}=z-\varepsilon u+Q(x) W(t), \\
\frac{d z}{d t}=-\Lambda z+\varepsilon z-\Phi u+\varepsilon \Lambda u-\varepsilon^{2} u-f(u)-\Lambda Q(x) W(t)+\varepsilon Q(x) W(t), \\
u(x, \tau)=u_{0}(x), \quad z(\tau, \omega)=z(x, \tau, \omega)=u_{1}(x)+\varepsilon u_{0}(x)-Q(x) W(\tau), \quad x \in U .
\end{array}\right.
$$

In contrast to the stochastic differential equation (3.2), no stochastic differential appears here. Let $\varphi=\left(\varphi_{1}, \varphi_{2}\right)=\left(\left(u_{1}, z_{1}\right)^{T},\left(u_{2}, z_{2}\right)^{T}\right)$. Hence equation (3.4) can be written as

$$
\left\{\begin{array}{lll}
\dot{\varphi}_{1 t}+L_{1} \varphi_{1}=F_{1}\left(\varphi_{1}, \omega\right), & \varphi_{1}(\tau, \omega)=\left(u_{10}, z_{1}(\tau, \omega)\right)^{T}, & t \geq \tau, \\
\dot{\varphi}_{2 t}+L_{2} \varphi_{2}=F_{2}\left(\varphi_{2}, \omega\right), & \varphi_{2}(\tau, \omega)=\left(u_{20}, z_{2}(\tau, \omega)\right)^{T}, & t \geq \tau
\end{array}\right.
$$

where

$$
\begin{gathered}
L_{1}=\left(\begin{array}{cc}
\varepsilon I & -I \\
\alpha A-\varepsilon\left(\delta_{1}-\varepsilon\right) I & \left(\delta_{1}-\varepsilon\right) I
\end{array}\right), \quad L_{2}=\left(\begin{array}{cc}
\varepsilon I & -I \\
\beta A^{\frac{1}{2}}-\varepsilon\left(\delta_{2}-\varepsilon\right) I & \left(\delta_{2}-\varepsilon\right) I
\end{array}\right), \\
F_{1}\left(\varphi_{1}, \omega\right)=\left(\begin{array}{c}
q_{B}(x) W_{1}(t) \\
-k\left(u_{1}-u_{2}\right)^{+}+\left(p-\left\|u_{1}\right\|_{1}^{2}\right) A^{\frac{1}{2}} u_{1}-f_{B}\left(u_{1}\right)-\left(\delta_{1}-\varepsilon\right) q_{B}(x) W_{1}(t)
\end{array}\right),
\end{gathered}
$$

and

$$
F_{2}\left(\varphi_{2}, \omega\right)=\left(\begin{array}{c}
q_{S}(x) W_{2}(t) \\
k\left(u_{1}-u_{2}\right)^{+}-f_{S}\left(u_{2}\right)-\left(\delta_{2}-\varepsilon\right) q_{S}(x) W_{2}(t)
\end{array}\right) .
$$

We know from [31] that $L_{1}, L_{2}$ are the infinitesimal generators of $C_{0}$-semigroup $e^{L_{1} t}, e^{L_{2} t}$ on $V_{2}, V_{1}$, respectively. It is not difficult to check that the functions $F(\cdot, \omega)=$ $\left(F_{1}(\cdot, \omega), F_{2}(\cdot, \omega)\right): E \mapsto E$ are locally Lipschitz continuous with respect to $\varphi=\left(\varphi_{1}, \varphi_{2}\right)$ and bounded for every $\omega \in \Omega$. Thus, by the classical semigroup theory on the local existence and uniqueness of solutions of evolution differential equations in [31], we have the following theorems.

Theorem 3.1 Let (1.4)-(1.6) hold, $q_{B}(x) \in H^{3}(U) \cap H_{0}^{2}(U), q_{S}(x) \in H^{2}(U) \cap H_{0}^{1}(U)$, then for any $T>0, \tau \in \mathbb{R}, \omega \in \Omega$, and $\varphi(\tau, \omega)=\left(\varphi_{1}(\tau, \omega), \varphi_{2}(\tau, \omega)\right) \in E, R P D E(3.5)$ has a unique solution $\varphi(\cdot, \omega) \in C([\tau, \tau+T)$; E) in mild sense, i.e.,

$$
\left\{\begin{array}{l}
\varphi_{1}(t, \omega)=e^{L_{1}(t-\tau)} \varphi_{1}(\tau, \omega)+\int_{\tau}^{t} e^{L_{1}(t-s)} F_{1}\left(\varphi_{1}(s), \omega\right) d s \\
\varphi_{2}(t, \omega)=e^{L_{2}(t-\tau)} \varphi_{2}(\tau, \omega)+\int_{\tau}^{t} e^{L_{2}(t-s)} F_{2}\left(\varphi_{2}(s), \omega\right) d s
\end{array}\right.
$$

Moreover, $\varphi(t, \varphi(\tau, \omega))$ is continuous in $t$ and $\varphi(\tau, \omega)$. 
Theorem 3.2 Let (1.4)-(1.6) hold, $q_{B}(x) \in H^{3}(U) \cap H_{0}^{2}(U), q_{S}(x) \in H^{2}(U) \cap H_{0}^{1}(U)$, then the solution $\varphi(\cdot, \omega) \in C([\tau, \tau+T)$;E) of system (3.5) generates a continuous random dynamical system $\left(\theta, S_{\varepsilon}(t, \omega)\right)$ on $E$ as

$$
S_{\varepsilon}(t, \omega): \varphi(\tau, \omega) \mapsto \varphi(t, \omega), \quad E \rightarrow E,
$$

by $S_{\varepsilon}(t, \omega)=\varphi(t+\tau, \tau, \varphi(\tau, \omega))=\left(\varphi_{1}(t+\tau, \tau, \varphi(\tau, \omega)), \varphi_{2}(t+\tau, \tau, \varphi(\tau, \omega))\right)$, where

$$
\begin{aligned}
& \varphi_{1}(t+\tau, \tau, \varphi(\tau, \omega))=\left(\begin{array}{c}
u_{1}(t+\tau, \tau, \varphi(\tau, \omega)) \\
u_{1 t}(t+\tau, \tau, \varphi(\tau, \omega))+\varepsilon u_{1}(t+\tau, \tau, \varphi(\tau, \omega))-q_{B}(x) W_{1}(t)
\end{array}\right) \\
& \varphi_{2}(t+\tau, \tau, \varphi(\tau, \omega))=\left(\begin{array}{c}
u_{2}(t+\tau, \tau, \varphi(\tau, \omega)) \\
u_{2 t}(t+\tau, \tau, \varphi(\tau, \omega))+\varepsilon u_{2}(t+\tau, \tau, \varphi(\tau, \omega))-q_{S}(x) W_{2}(t)
\end{array}\right) .
\end{aligned}
$$

To show the conjugation of the solution of the stochastic partial differential equation (3.2) and RPDE (3.5), we introduced the homeomorphism

$$
R\left(\theta_{t} \omega\right) y=\left(y_{1}, y_{2}-\varepsilon y_{1}+Q(x) W(t)\right)^{T}, \quad y=\left(y_{1}, y_{2}\right)^{T} \in E
$$

with the inverse homeomorphism being

$$
R^{-1}\left(\theta_{t} \omega\right) y=\left(y_{1}, y_{2}+\varepsilon y_{1}-Q(x) W(t)\right)^{T} .
$$

Then the transformation

$$
S(t, \omega)=R\left(\theta_{t} \omega\right) S_{\varepsilon}(t, \omega) R^{-1}\left(\theta_{t} \omega\right)
$$

also determines an RDS corresponding to equation (3.2).

We will also use the transformation, let

$$
\begin{aligned}
& \eta(t)=\left(\eta_{1}(t), \eta_{2}(t)\right)=\left(\left(u_{1}(t), u_{1 t}(t)+\varepsilon u_{1}(t)\right)^{T},\left(u_{2}(t), u_{2 t}(t)+\varepsilon u_{2}(t)\right)^{T}\right), \\
& 0 \\
& G_{1}\left(\eta_{1}, \omega\right)=\left(\begin{array}{c} 
\\
-k\left(u_{1}-u_{2}\right)^{+}+\left(p-\left\|u_{1}\right\|_{1}^{2}\right) A^{\frac{1}{2}} u_{1}-f_{B}\left(u_{1}\right)+q_{B}(x) \dot{W}_{1}(t)
\end{array}\right),
\end{aligned}
$$

and

$$
G_{2}\left(\eta_{2}, \omega\right)=\left(\begin{array}{c}
0 \\
k\left(u_{1}-u_{2}\right)^{+}-f_{S}\left(u_{2}\right)+q_{S}(x) \dot{W}_{2}(t)
\end{array}\right),
$$

then RPDE (3.2) can be rewritten as

$$
\begin{cases}\eta_{1 t}+L_{1} \eta_{1}=G_{1}\left(\eta_{1}, \omega\right), & \eta_{1}(\tau, \omega)=\left(u_{10}, u_{11}+\varepsilon u_{10}\right)^{T}, \\ \eta_{2 t}+L_{2} \eta_{2}=G_{2}\left(\eta_{2}, \omega\right), & \eta_{2}(\tau, \omega)=\left(u_{20}, u_{21}+\varepsilon u_{20}\right)^{T} .\end{cases}
$$

We introduce the isomorphism $T_{\varepsilon} y=\left(y_{1}, y_{2}+\varepsilon y_{1}\right)^{T}, y=\left(y_{1}, y_{2}\right)^{T} \in E$, which has the inverse isomorphism $T_{-\varepsilon} y=\left(y_{1}, y_{2}-\varepsilon y_{1}\right)^{T}$. It follows that $\left(\theta, \bar{S}_{\varepsilon}(t, \omega)\right)$ with the transformation

$$
\bar{S}_{\varepsilon}(t, \omega)=T_{\varepsilon} S(t, \omega) T_{-\varepsilon}
$$


is also an RDS corresponding to (3.5). Therefore, the two $\operatorname{RDS} S(t, \omega)$ and $\bar{S}_{\varepsilon}(t, \omega)$ are equivalent.

\section{Existence of a random attractor}

In this section, we prove the existence of a random attractor for RDS (3.5) in the space $E$.

Lemma 4.1 Let (1.4)-(1.6) hold, $q_{B}(x) \in H^{3}(U) \cap H_{0}^{2}(U), q_{S}(x) \in H^{2}(U) \cap H_{0}^{1}(U)$. There exist a random variable $r_{1}(\omega)>0$ and a bounded ball $B_{0}$ of $E$ centered at 0 with random radius $r_{0}(\omega)>0$ such that, for any bounded non-random set $B$ of $E$, there exists a deterministic $T(B) \leq-1$ such that the solution $\varphi(t, \omega ; \varphi(\tau, \omega))=\left(\varphi_{1}\left(t, \omega ; \varphi_{1}(\tau, \omega)\right), \varphi_{2}\left(t, \omega ; \varphi_{2}(\tau, \omega)\right)\right)=$ $\left(\left(u_{1}(t, \omega), z_{1}(t, \omega)\right)^{T},\left(u_{2}(t, \omega), z_{2}(t, \omega)\right)^{T}\right)$ of (3.5) with the initial value $\left(\left(u_{10}, u_{11}+\varepsilon u_{10}\right)^{T}\right.$, $\left.\left(u_{20}, u_{21}+\varepsilon u_{20}\right)^{T}\right) \in B$ satisfies, for P-a.s. $\omega \in \Omega$,

$$
\|\varphi(-1, \omega ; \varphi(\tau, \omega))\|_{E} \leq r_{0}(\omega), \quad \tau \leq T(B)
$$

and for all $\tau \leq t \leq 0$,

$$
\|\varphi(t, \omega ; \varphi(\tau, \omega))\|_{E}^{2} \leq R_{1}(t, \omega)
$$

where $z_{1}(t, \omega)=u_{1 t}(t)+\varepsilon u_{1}(t)-q_{B}(x) W_{1}(t), z_{2}(t, \omega)=u_{2 t}(t)+\varepsilon u_{2}(t)-q_{S}(x) W_{2}(t)$, and $R(t, \omega)$ is given by

$$
\begin{aligned}
R_{1}(t, \omega)= & e^{-\varepsilon_{1}(t-\tau)}\left(\left\|u_{10}\right\|_{2}^{2}+\left\|u_{11}+\varepsilon u_{10}\right\|^{2}+\left\|q_{B}\right\|^{2}\left|W_{1}(\tau)\right|^{2}+\left\|u_{20}\right\|_{1}^{2}+\left\|u_{21}+\varepsilon u_{20}\right\|^{2}\right. \\
& +\left\|q_{S}\right\|^{2}\left|W_{2}(\tau)\right|^{2}+\frac{1}{2}\left(\left\|u_{10}\right\|_{1}^{2}-p\right)^{2}+k\left\|\left(u_{10}-u_{20}\right)^{+}\right\|^{2} \\
& \left.\left.+2 \int_{\Omega} F_{B}\left(u_{10}\right) d x+2 \int_{\Omega} F_{S}\left(u_{20}\right) d x\right)+M r_{1}^{2}(\omega)\right) .
\end{aligned}
$$

It is easy to deduce a similar absorption result for

$$
\eta(-1)=\left(\eta_{1}, \eta_{2}\right)=\left(\left(u_{1}(-1), u_{1 t}(-1)+\varepsilon u_{1}(-1)\right)^{T},\left(u_{2}(-1), u_{2 t}(-1)+\varepsilon u_{2}(-1)\right)^{T}\right)
$$

instead of $\varphi(-1)$.

Proof Taking the inner product in $V_{2}$ of the first equation of (3.5) with $\varphi_{1}=\left(u_{1}, z_{1}\right)^{T}$, and taking the inner product in $V_{1}$ of the second equation of (3.5) with $\varphi_{2}=\left(u_{2}, z_{2}\right)^{T}$, in which $z_{1}=u_{1 t}+\varepsilon u_{1}-q_{B}(x) W_{1}(t), z_{2}=u_{2 t}+\varepsilon u_{2}-q_{S}(x) W_{2}(t)$, then adding them, we find that

$$
\begin{gathered}
\frac{1}{2} \frac{d}{d t}\left\|\varphi_{1}\right\|_{V_{2}}^{2}+\left(L_{1} \varphi_{1}, \varphi_{1}\right)_{V_{2}}+\frac{1}{2} \frac{d}{d t}\left\|\varphi_{2}\right\|_{V_{1}}^{2}+\left(L_{2} \varphi_{2}, \varphi_{2}\right)_{V_{1}} \\
\quad=\left(F_{1}\left(\varphi_{1}, \omega\right), \varphi_{1}\right)_{V_{2}}+\left(F_{2}\left(\varphi_{2}, \omega\right), \varphi_{2}\right)_{V_{1}}, \quad \forall t \geq \tau .
\end{gathered}
$$

Due to (3.1), using the Hölder and Young inequalities, we get

$$
\begin{aligned}
& \left(L_{1} \varphi_{1}, \varphi_{1}\right)_{V_{2}}+\left(L_{2} \varphi_{2}, \varphi_{2}\right)_{V_{1}} \\
& \quad=\varepsilon\left\|u_{1}\right\|_{2}^{2}+(\alpha-1)\left(A u_{1}, z_{1}\right)-\varepsilon\left(\delta_{1}-\varepsilon\right)\left(u_{1}, z_{1}\right)+\left(\delta_{1}-\varepsilon\right)\left\|z_{1}\right\|^{2}
\end{aligned}
$$




$$
\begin{aligned}
& +\varepsilon\left\|u_{2}\right\|_{1}^{2}+(\beta-1)\left(A^{\frac{1}{2}} u_{2}, z_{2}\right)-\varepsilon\left(\delta_{2}-\varepsilon\right)\left(u_{2}, z_{2}\right)+\left(\delta_{2}-\varepsilon\right)\left\|z_{2}\right\|^{2} \\
\geq & \frac{1}{2} \frac{d}{d t}\left((\alpha-1)\left\|u_{1}\right\|_{2}^{2}+(\beta-1)\left\|u_{2}\right\|_{1}^{2}\right)+\varepsilon\left(\alpha\left\|u_{1}\right\|_{2}^{2}+\beta\left\|u_{2}\right\|_{1}^{2}\right) \\
& -\frac{\varepsilon \delta_{1}}{\sqrt{\lambda_{1}}}\left\|u_{1}\right\|_{2}\left\|z_{1}\right\|+\left(\delta_{1}-\varepsilon\right)\left\|z_{1}\right\|^{2}-\frac{\varepsilon \delta_{2}}{\sqrt[4]{\lambda_{1}}}\left\|u_{2}\right\|_{1}\left\|z_{2}\right\|+\left(\delta_{2}-\varepsilon\right)\left\|z_{2}\right\|^{2} \\
& +(1-\alpha)\left(A u_{1}, q_{B}(x) W_{1}(t)\right)+(1-\beta)\left(A^{\frac{1}{2}} u_{2}, q_{S}(x) W_{2}(t)\right) \\
\geq & \frac{1}{2} \frac{d}{d t}\left((\alpha-1)\left\|u_{1}\right\|_{2}^{2}+(\beta-1)\left\|u_{2}\right\|_{1}^{2}\right)+\varepsilon\left(\alpha\left\|u_{1}\right\|_{2}^{2}+\beta\left\|u_{2}\right\|_{1}^{2}\right)-\frac{\varepsilon \alpha}{4}\left\|u_{1}\right\|_{2}^{2} \\
& -\frac{\varepsilon \delta_{1}^{2}}{\alpha \lambda_{1}}\left\|z_{1}\right\|^{2}+\left(\delta_{1}-\varepsilon\right)\left\|z_{1}\right\|^{2}-\frac{\varepsilon \beta}{4}\left\|u_{2}\right\|_{1}^{2}-\frac{\varepsilon \delta_{2}^{2}}{\beta \sqrt{\lambda_{1}}}\left\|z_{2}\right\|^{2}+\left(\delta_{2}-\varepsilon\right)\left\|z_{2}\right\|^{2} \\
& +(1-\alpha)\left(A u_{1}, q_{B}(x) W_{1}(t)\right)+(1-\beta)\left(A^{\frac{1}{2}} u_{2}, q_{S}(x) W_{2}(t)\right) \\
\geq & \frac{1}{2} \frac{d}{d t}\left((\alpha-1)\left\|u_{1}\right\|_{2}^{2}+(\beta-1)\left\|u_{2}\right\|_{1}^{2}\right)+\frac{\varepsilon}{2}\|\varphi\|_{E}^{2}+\frac{\varepsilon}{4}\left(\alpha\left\|u_{1}\right\|_{2}^{2}+\beta\left\|u_{2}\right\|_{1}^{2}\right) \\
& +\frac{\delta_{1}}{2}\left\|z_{1}\right\|^{2}+\frac{\delta_{2}}{2}\left\|z_{2}\right\|^{2}+(1-\alpha)\left(A u_{1}, q_{B}(x) W_{1}(t)\right) \\
& +(1-\beta)\left(A u^{\frac{1}{2}} u_{2}, q_{S}(x) W_{2}(t)\right),
\end{aligned}
$$

noting that we use (3.3) in the last inequality. In addition,

$$
\begin{aligned}
\left(F_{1}\left(\varphi_{1}, \omega\right), \varphi_{1}\right)_{V_{2}}+\left(F_{2}\left(\varphi_{2}, \omega\right), \varphi_{2}\right)_{V_{1}} \\
=\left(u_{1}, q_{B}(x) W_{1}(t)\right)_{2}+\left(u_{2}, q_{S}(x) W_{2}(t)\right)_{1}-\left(k\left(u_{1}-u_{2}\right)^{+}, z_{1}\right)+\left(k\left(u_{1}-u_{2}\right)^{+}, z_{2}\right) \\
\quad-\left(f_{B}\left(u_{1}\right), z_{1}\right)+\left(\left(p-\left\|u_{1}\right\|_{1}^{2}\right) A^{\frac{1}{2}} u_{1}, z_{1}\right)-\left(\delta_{1}-\varepsilon\right)\left(q_{B}(x) W_{1}(t), z_{1}\right)-\left(f_{S}\left(u_{2}\right), z_{2}\right) \\
\quad-\left(\delta_{2}-\varepsilon\right)\left(q_{S}(x) W_{2}(t), z_{2}\right) \\
=-\frac{1}{2} \frac{d}{d t} k\left\|\left(u_{1}-u_{2}\right)^{+}\right\|^{2}-k \varepsilon\left\|\left(u_{1}-u_{2}\right)^{+}\right\|^{2}+\left(u_{1}, q_{B}(x) W_{1}(t)\right)_{2}+\left(u_{2}, q_{S}(x) W_{2}(t)\right)_{1} \\
\quad+k\left(\left(u_{1}-u_{2}\right)^{+}, q_{B}(x) W_{1}(t)\right)-k\left(\left(u_{1}-u_{2}\right)^{+}, q_{S}(x) W_{2}(t)\right)-\left(\delta_{1}-\varepsilon\right)\left(q_{B}(x) W_{1}(t), z_{1}\right) \\
\quad-\left(\delta_{2}-\varepsilon\right)\left(q_{S}(x) W_{2}(t), z_{2}\right)+\left(\left(p-\left\|u_{1}\right\|_{1}^{2}\right) A^{\frac{1}{2}} u_{1}, z_{1}\right) \\
\quad-\left(f_{B}\left(u_{1}\right), z_{1}\right)-\left(f_{S}\left(u_{2}\right), z_{2}\right) .
\end{aligned}
$$

We deal with some terms in (4.3) and (4.4) as follows:

$$
\begin{aligned}
& \alpha\left(u_{1}, q_{B}(x) W_{1}(t)\right)_{2}+\beta\left(u_{2}, q_{S}(x) W_{2}(t)\right)_{1} \\
& \quad \leq \frac{\varepsilon}{4}\left(\alpha\left\|u_{1}\right\|_{2}^{2}+\beta\left\|u_{2}\right\|_{1}^{2}\right)+\frac{\alpha}{\varepsilon}\left\|q_{B}\right\|_{2}^{2}\left|W_{1}(t)\right|^{2}+\frac{\beta}{\varepsilon}\left\|q_{S}\right\|_{1}^{2}\left|W_{2}(t)\right|^{2} ; \\
& \left|k\left(\left(u_{1}-u_{2}\right)^{+}, q_{B}(x) W_{1}(t)\right)-k\left(\left(u_{1}-u_{2}\right)^{+}, q_{S}(x) W_{2}(t)\right)\right| \\
& \quad \leq \frac{k \varepsilon}{2}\left\|\left(u_{1}-u_{2}\right)^{+}\right\|^{2}+\frac{k}{\varepsilon}\left\|q_{B}\right\|^{2}\left|W_{1}(t)\right|^{2}+\frac{k}{\varepsilon}\left\|q_{S}\right\|^{2}\left|W_{2}(t)\right|^{2} ; \\
& \left|-\left(\delta_{1}-\varepsilon\right)\left(q_{B}(x) W_{1}(t), z_{1}\right)-\left(\delta_{2}-\varepsilon\right)\left(q_{S}(x) W_{2}(t), z_{2}\right)\right| \\
& \quad \leq \frac{\delta_{1}}{2}\left\|z_{1}\right\|^{2}+\frac{\delta_{2}}{2}\left\|z_{2}\right\|^{2}+\frac{\delta_{1}}{2}\left\|q_{B}\right\|^{2}\left|W_{1}(t)\right|^{2}+\frac{\delta_{2}}{2}\left\|q_{S}\right\|^{2}\left|W_{2}(t)\right|^{2} .
\end{aligned}
$$


Furthermore, we get

$$
\begin{aligned}
- & \left(\left\|u_{1}\right\|_{1}^{2}-p\right)\left(A^{\frac{1}{2}} u_{1}, z_{1}\right) \\
= & -\frac{1}{4} \frac{d}{d t}\left(\left\|u_{1}\right\|_{1}^{2}-p\right)^{2}-\frac{\varepsilon}{2}\left(\left\|u_{1}\right\|_{1}^{2}-p\right)^{2}-\frac{\varepsilon}{2}\left\|u_{1}\right\|_{1}^{4}+\frac{\varepsilon p^{2}}{2} \\
& +\left(\left\|u_{1}\right\|_{1}^{2}-p\right)\left(A^{\frac{1}{2}} u_{1}, q_{B}(x) W_{1}(t)\right) \\
\leq & -\frac{1}{4} \frac{d}{d t}\left(\left\|u_{1}\right\|_{1}^{2}-p\right)^{2}-\frac{\varepsilon}{2}\left(\left\|u_{1}\right\|_{1}^{2}-p\right)^{2}-\frac{\varepsilon}{2}\left\|u_{1}\right\|_{1}^{4}+\frac{\varepsilon p^{2}}{2}+\frac{\varepsilon}{4}\left(\left\|u_{1}\right\|_{1}^{2}-p\right)^{2} \\
& +\frac{\varepsilon}{2}\left\|u_{1}\right\|_{1}^{4}+\frac{\left\|q_{B}\right\|_{1}^{4}}{2 \varepsilon^{3}}\left|W_{1}(t)\right|^{4} \\
= & -\frac{1}{4} \frac{d}{d t}\left(\left\|u_{1}\right\|_{1}^{2}-p\right)^{2}-\frac{\varepsilon}{4}\left(\left\|u_{1}\right\|_{1}^{2}-p\right)^{2}+\frac{\varepsilon p^{2}}{2}+\frac{\left\|q_{B}\right\|_{1}^{4}}{2 \varepsilon^{3}}\left|W_{1}(t)\right|^{4} .
\end{aligned}
$$

By means of (1.4) and (1.5), we conclude that

$$
\begin{aligned}
& \left(f_{B}\left(u_{1}\right), q_{B}(x) W_{1}(t)\right) \\
& \quad \leq C_{0} \int_{U}\left(1+\left|u_{1}\right|^{\gamma}\right) q_{B}(x) W_{1}(t) d x \\
& \quad \leq C_{0}\left\|q_{B}\right\|\left|W_{1}(t)\right|+C_{0}\left(\int_{U}\left|u_{1}\right|^{\gamma+1} d x\right)^{\frac{\gamma}{\gamma+1}}\left\|q_{B}\right\|_{\gamma+1}\left|W_{1}(t)\right| \\
& \quad \leq C_{0}\left\|q_{B}\right\|\left|W_{1}(t)\right|+C_{0} C_{1}^{-\frac{\gamma}{\gamma+1}}\left(\int_{U}\left(F_{B}\left(u_{1}\right)+C_{1}\right) d x\right)^{\frac{\gamma}{\gamma+1}}\left\|q_{B}\right\|_{\gamma+1}\left|W_{1}(t)\right| \\
& \quad \leq C_{0}\left\|q_{B}\right\|\left|W_{1}(t)\right|+\frac{\varepsilon C_{0} C_{1}^{-1}}{2} \int_{U} F_{B}\left(u_{1}\right) d x+\frac{C_{0}}{2 \varepsilon}\left\|q_{B}\right\|_{\gamma+1}^{\gamma+1}\left|W_{1}(t)\right|^{\gamma+1}+\frac{\varepsilon C_{0}|U|}{2}
\end{aligned}
$$

so together (1.6) with (4.9), this yields

$$
\begin{aligned}
- & \left(f_{B}\left(u_{1}\right), z_{1}\right) \\
= & -\left(f_{B}\left(u_{1}\right), u_{1 t}+\varepsilon u_{1}-q_{B} W_{1}(t)\right) \\
\leq & -\frac{d}{d t} \int_{U} F_{B}\left(u_{1}\right) d x-\varepsilon C_{2} \int_{U} F_{B}\left(u_{1}\right) d x+\varepsilon C_{2}|U|+\left(f_{B}\left(u_{1}\right), q_{B} W_{1}(t)\right) \\
\leq & -\frac{d}{d t} \int_{U} F_{B}\left(u_{1}\right) d x-\frac{\varepsilon\left(2 C_{2}-C_{0} C_{1}^{-1}\right)}{2} \int_{U} F_{B}\left(u_{1}\right) d x+C_{0}\left\|q_{B}\right\|\left|W_{1}(t)\right| \\
& +\frac{C_{0}}{2 \varepsilon}\left\|q_{B}\right\|_{\gamma+1}^{\gamma+1}\left|W_{1}(t)\right|^{\gamma+1}+\frac{\varepsilon\left(C_{0}+2 C_{2}\right)}{2}|U| .
\end{aligned}
$$

Similarly, there holds w

$$
\begin{aligned}
- & \left(f_{S}\left(u_{2}\right), z_{2}\right) \\
= & -\left(f_{S}\left(u_{2}\right), u_{2 t}+\varepsilon u_{2}-q_{2} W_{2}(t)\right) \\
\leq & -\frac{d}{d t} \int_{U} F_{S}\left(u_{2}\right) d x-\frac{\varepsilon\left(2 C_{2}-C_{0} C_{1}^{-1}\right)}{2} \int_{U} F_{S}\left(u_{2}\right) d x+C_{0}\left\|q_{S}\right\|\left|W_{2}(t)\right| \\
& +\frac{C_{0}}{2 \varepsilon}\left\|q_{S}\right\|_{\gamma+1}^{\gamma+1}\left|W_{2}(t)\right|^{\gamma+1}+\frac{\varepsilon\left(C_{0}+2 C_{2}\right)}{2}|U| .
\end{aligned}
$$


Therefore, collecting with (4.2)-(4.11), we get

$$
\begin{aligned}
& \frac{d}{d t}\left(\|\varphi\|_{E}^{2}+\frac{1}{2}\left(\left\|u_{1}\right\|_{1}^{2}-p\right)^{2}+k\left\|\left(u_{1}-u_{2}\right)^{+}\right\|^{2}+2 \int_{U} F_{B}\left(u_{1}\right) d x\right. \\
& \left.\quad+2 \int_{U} F_{S}\left(u_{2}\right) d x+4 C_{1}|U|\right)+\varepsilon\left(\|\varphi\|_{E}^{2}\right. \\
& \quad+\frac{1}{2}\left(\left\|u_{1}\right\|_{1}^{2}-p\right)^{2}+k\left\|\left(u_{1}-u_{2}\right)^{+}\right\|^{2}+\left(2 C_{2}-C_{0} C_{1}^{-1}\right) \int_{U} F_{B}\left(u_{1}\right) d x \\
& \left.\quad+\left(2 C_{2}-C_{0} C_{1}^{-1}\right) \int_{U} F_{S}\left(u_{2}\right) d x+4 C_{1}|U|\right) \\
& \leq M\left(1+\left|W_{1}(t)\right|+\left|W_{1}(t)\right|^{2}+\left|W_{1}(t)\right|^{4}+\left|W_{1}(t)\right|^{\gamma+1}\right. \\
& \left.\quad+\left|W_{2}(t)\right|+\left|W_{2}(t)\right|^{2}+\left|W_{2}(t)\right|^{\gamma+1}\right) \\
& :=M(1+E(t))
\end{aligned}
$$

where $M=\max \left\{2 \varepsilon\left(C_{0}+2 C_{2}+2 C_{1}\right)|U|+\varepsilon p^{2}, 2 C_{0}\left\|q_{B}\right\|, \frac{2 \alpha}{\varepsilon}\left\|q_{B}\right\|_{2}^{2}+\left(\delta_{1}+\frac{2 k}{\varepsilon}\right)\left\|q_{B}\right\|^{2}, \frac{\left\|q_{B}\right\|_{1}^{4}}{\varepsilon^{3}}\right.$, $\left.\frac{C_{0}}{\varepsilon}\left\|q_{B}\right\|_{\gamma+1}^{\gamma+1}, 2 C_{0}\left\|q_{S}\right\|, \frac{2 \beta}{\varepsilon}\left\|q_{S}\right\|_{2}^{2}+\left(\delta_{2}+\frac{2 k}{\varepsilon}\right)\left\|q_{S}\right\|^{2}, \frac{C_{0}}{\varepsilon}\left\|q_{S}\right\|_{\gamma+1}^{\gamma+1}\right\}$. Using (1.5), we have the fact $2 \int_{U} F_{1}\left(u_{1}\right) d x+2 \int_{U} F_{2}\left(u_{2}\right) d x+4 C_{1}|U| \geq 0$, let

$$
\begin{aligned}
I(t)= & \|\varphi\|_{E}^{2}+\frac{1}{2}\left(\left\|u_{1}\right\|_{1}^{2}-p\right)^{2}+k\left\|\left(u_{1}-u_{2}\right)^{+}\right\|^{2}+2 \int_{U} F_{1}\left(u_{1}\right) d x+2 \int_{U} F_{2}\left(u_{2}\right) d x \\
& +4 C_{1}|U| \geq 0 .
\end{aligned}
$$

Choosing $\varepsilon_{1}=\min \left\{\varepsilon, \frac{\varepsilon\left(2 C_{2}-C_{0} C_{1}^{-1}\right)}{2}\right\}$ and $C_{2}>\frac{C_{0} C_{1}^{-1}}{2}$, then we have that

$$
\frac{d}{d t} I(t)+\varepsilon_{1} I(t) \leq M(1+E(t))
$$

By the Gronwall lemma, we conclude that

$$
\begin{aligned}
& \|\varphi(t, \omega ; \varphi(\tau, \omega))\|_{E}^{2} \\
& \leq e^{-\varepsilon_{1}(t-\tau)} I(\tau)+M \int_{\tau}^{t} e^{-\varepsilon_{1}(t-s)}(1+E(s)) d s \\
& =e^{-\varepsilon_{1}(t-\tau)}\left(\left\|u_{10}\right\|_{2}^{2}+\left\|u_{11}+\varepsilon u_{10}\right\|^{2}+\left\|q_{B}\right\|^{2}\left|W_{1}(\tau)\right|^{2}+\left\|u_{20}\right\|_{1}^{2}+\left\|u_{21}+\varepsilon u_{20}\right\|^{2}\right. \\
& \quad+\left\|q_{S}\right\|^{2}\left|W_{2}(\tau)\right|^{2}+\frac{1}{2}\left(\left\|u_{10}\right\|_{1}^{2}-p\right)^{2}+k\left\|\left(u_{10}-u_{20}\right)^{+}\right\|^{2}+2 \int_{U} F_{B}\left(u_{10}\right) d x \\
& \left.\quad+2 \int_{U} F_{S}\left(u_{20}\right) d x+4 C_{1}|U|\right)+M \int_{\tau}^{t} e^{-\varepsilon_{1}(t-s)}(1+E(s)) d s .
\end{aligned}
$$

Take

$$
r_{0}^{2}(\omega)=1+\frac{M}{\varepsilon_{1}}+\sup _{\tau \leq-1} e^{\varepsilon_{1} \tau}\left(\left\|q_{B}\right\|^{2}\left|W_{1}(\tau)\right|^{2}+\left\|q_{S}\right\|^{2}\left|W_{2}(\tau)\right|^{2}\right)+M \int_{-\infty}^{-1} e^{-\varepsilon_{1}(-1-s)} E(s) d s
$$


and

$$
r_{1}^{2}(\omega)=\frac{1}{\varepsilon_{1}}+\int_{-\infty}^{0} e^{\varepsilon_{1} s} E(s) d s
$$

Since $\lim _{t \rightarrow \infty} \frac{W_{1}(t)}{t}=0, \lim _{t \rightarrow \infty} \frac{W_{2}(t)}{t}=0, r_{0}^{2}(\omega)$ and $r_{1}^{2}(\omega)$ are finite $P$-a.s. Given a bounded set $B$ of $E$, choose $T(B) \leq-1$ such that

$$
\begin{gathered}
e^{-\varepsilon_{1}(-1-\tau)}\left(\left\|u_{10}\right\|_{2}^{2}+\left\|u_{11}+\varepsilon u_{10}\right\|^{2}+\left\|u_{20}\right\|_{1}^{2}+\left\|u_{21}+\varepsilon u_{20}\right\|^{2}+\frac{1}{2}\left(\left\|u_{10}\right\|_{1}^{2}-p\right)^{2}\right. \\
\left.+k\left\|\left(u_{10}-u_{20}\right)^{+}\right\|^{2}+2 \int_{U} F_{B}\left(u_{10}\right) d x+2 \int_{U} F_{S}\left(u_{20}\right) d x+4 C_{1}|U|\right) \leq 1
\end{gathered}
$$

for all $\left(\left(u_{10}, u_{11}+\varepsilon u_{10}\right)^{T},\left(u_{20}, u_{21}+\varepsilon u_{20}\right)^{T}\right) \in B$, and for all $\tau \leq T(B)$.

This completes the proof of Lemma 4.1.

In order to obtain the regularity estimates later, we decompose the solution $u(t)=$ $\left(u_{1}(t), u_{2}(t)\right)$ of system $(1.1)-(1.3)$ with the initial value $\left(\left(u_{10}, u_{11}+\varepsilon u_{10}\right)^{T},\left(u_{20}, u_{21}+\right.\right.$ $\left.\left.\varepsilon u_{20}\right)^{T}\right) \in B$ into two parts $u_{1}(t)=y_{11}(t)+y_{12}(t), u_{2}(t)=y_{21}(t)+y_{22}(t)$, where $\left(y_{11}(t), y_{21}(t)\right)$, $\left(y_{12}(t), y_{22}(t)\right)$ satisfy

$$
\left\{\begin{array}{l}
y_{11 t t}+\alpha \triangle^{2} y_{11}+\delta_{1} y_{11 t}+\left(p-\|u\|_{1}^{2}\right) \triangle y_{11}=0, \quad \text { in } U \times[\tau,+\infty), \tau \in \mathbb{R}, \\
y_{21 t t}-\beta \triangle y_{21}+\delta_{2} y_{21 t}=0, \quad \text { in } U \times[\tau,+\infty), \tau \in \mathbb{R} \\
y_{11}(x, t)=y_{11}(x, t)=y_{21}(x, t)=0, \quad x \in \partial U, t \geq \tau \\
y_{11}(x, \tau)=u_{10}(x), \quad y_{11 t}(x, \tau)=u_{11}(x), \quad x \in U \\
y_{21}(x, \tau)=u_{20}(x), \quad y_{21 t}(x, \tau)=u_{21}(x), \quad x \in U
\end{array}\right.
$$

and

$$
\left\{\begin{array}{c}
y_{12 t t}+\alpha \Delta^{2} y_{12}+\delta_{1} y_{12 t}+\left(p-\|u\|_{1}^{2}\right) \Delta y_{12}+k\left(u_{1}-u_{2}\right)^{+} \\
+f_{B}\left(u_{1}\right)=q_{B}(x) \dot{W}_{1}(t), \quad \text { in } U \times[\tau,+\infty), \tau \in \mathbb{R}, \\
y_{22 t t}-\beta \Delta y_{22}+\delta_{2} y_{22 t}-k\left(u_{1}-u_{2}\right)^{+} \\
+f_{S}\left(u_{2}\right)=q_{S}(x) \dot{W}_{2}(t), \quad \text { in } U \times[\tau,+\infty), \tau \in \mathbb{R}, \\
y_{12}(x, t)=\Delta y_{12}(x, t)=y_{22}(x, t)=0, \quad x \in \partial U, t \geq \tau, \\
y_{12}(x, \tau)=0, \quad y_{12 t}(x, \tau)=0, \quad x \in U, \\
y_{22}(x, \tau)=0, \quad y_{22 t}(x, \tau)=0, \quad x \in U .
\end{array}\right.
$$

Lemma 4.2 Assume that $p \leq \frac{\alpha \sqrt{\lambda_{1}}}{3}, B$ is a bounded non-random subset of $E$, then for any $\left(\left(u_{10}, u_{11}+\varepsilon u_{10}\right)^{T},\left(u_{20}, u_{21}+\varepsilon u_{20}\right)^{T}\right) \in B$,

$$
\begin{aligned}
\left\|Y_{1}(0)\right\|_{E}^{2}= & \alpha\left\|y_{11}(0)\right\|_{2}^{2}+\left\|y_{11 t}(0)+\varepsilon y_{11}(0)\right\|^{2}+\beta\left\|y_{21}(0)\right\|_{1}^{2}+\left\|y_{21 t}(0)+\varepsilon y_{21}(0)\right\|^{2} \\
\leq & \frac{e^{\frac{\varepsilon \tau}{2}}}{C(p)}\left(\alpha\left\|u_{10}\right\|_{2}^{2}-p\left\|u_{10}\right\|_{1}^{2}+\left\|u_{11}+\varepsilon u_{10}\right\|^{2}+\beta\left\|u_{20}\right\|_{1}^{2}\right. \\
& \left.+\left\|u_{21}+\varepsilon u_{20}\right\|^{2}+\left\|u_{10}\right\|_{1}^{4}\right),
\end{aligned}
$$


where $Y_{1}=\left(\left(y_{11}, y_{11 t}+\varepsilon y_{11}\right)^{T},\left(y_{21}, y_{21 t}+\varepsilon y_{21}\right)^{T}\right)$ satisfies (4.15), and

$$
C(p)= \begin{cases}1, & p \leq 0 \\ 1-\frac{p}{\alpha \sqrt{\lambda_{1}}}, & 0<p \leq \frac{\alpha \sqrt{\lambda_{1}}}{3}\end{cases}
$$

Proof Taking the scalar product in $L^{2}(U)$ of the first and second equation of (4.15) with $v_{1}=y_{11 t}+\varepsilon y_{11}$ and $v_{2}=y_{21 t}+\varepsilon y_{21}$, respectively, then adding them, we conclude that

$$
\begin{aligned}
& \frac{1}{2} \frac{d}{d t}\left(\alpha\left\|y_{11}\right\|_{2}^{2}+\left\|v_{1}\right\|^{2}+\beta\left\|y_{21}\right\|_{1}^{2}+\left\|v_{2}\right\|^{2}\right)+\left(p-\|u\|_{1}^{2}\right)\left(A^{\frac{1}{2}} y_{11}, v_{1}\right)+\varepsilon \alpha\left\|y_{11}\right\|_{2}^{2} \\
& \quad+\left(\delta_{1}-\varepsilon\right)\left\|v_{1}\right\|^{2}-\varepsilon\left(\delta_{1}-\varepsilon\right)\left(y_{11}, v_{1}\right)+\varepsilon \beta\left\|y_{21}\right\|_{1}^{2}+\left(\delta_{2}-\varepsilon\right)\left\|v_{2}\right\|^{2} \\
& \quad-\varepsilon\left(\delta_{2}-\varepsilon\right)\left(y_{21}, v_{2}\right)=0 .
\end{aligned}
$$

It is easy to derive that

$$
\begin{aligned}
& \left(\|u\|_{1}^{2}-p\right)\left(A^{\frac{1}{2}} y_{11}, v_{1}\right) \\
& \quad=\frac{1}{2} \frac{d}{d t}\left(\|u\|_{1}^{2}\left\|y_{11}\right\|_{1}^{2}-p\left\|u_{1}\right\|_{1}^{2}\right)+\varepsilon\left(\|u\|_{1}^{2}\left\|y_{11}\right\|_{1}^{2}-p\left\|y_{11}\right\|_{1}^{2}\right) .
\end{aligned}
$$

Due to (3.3), we derive that $0<\varepsilon<\min \left\{\frac{\delta_{1}}{3}, \frac{\alpha \lambda_{1}}{2 \delta_{1}}, \frac{\delta 2}{3}, \frac{\beta \sqrt{\lambda_{1}}}{2 \delta_{2}}\right\}$, then it follows that

$$
\begin{aligned}
& \varepsilon \alpha\left\|y_{11}\right\|_{2}^{2}+\left(\delta_{1}-\varepsilon\right)\left\|v_{1}\right\|^{2}-\varepsilon\left(\delta_{1}-\varepsilon\right)\left(y_{11}, v_{1}\right) \\
& +\varepsilon \beta\left\|y_{21}\right\|_{1}^{2}+\left(\delta_{2}-\varepsilon\right)\left\|v_{2}\right\|^{2}-\varepsilon\left(\delta_{2}-\varepsilon\right)\left(y_{21}, v_{2}\right) \\
& \geq \varepsilon \alpha\left\|y_{11}\right\|_{2}^{2}+\frac{2 \delta_{1}}{3}\left\|v_{1}\right\|^{2}-\frac{\varepsilon \alpha}{2}\left\|y_{11}\right\|_{2}^{2}-\frac{\varepsilon \delta_{1}^{2}}{2 \alpha \lambda_{1}}\left\|v_{1}\right\|^{2} \\
& \quad+\varepsilon \beta\left\|y_{21}\right\|_{1}^{2}+\frac{2 \delta_{2}}{3}\left\|v_{2}\right\|^{2}-\frac{\varepsilon \beta}{2}\left\|y_{21}\right\|_{2}^{2}-\frac{\varepsilon \delta_{2}^{2}}{2 \beta \sqrt{\lambda_{1}}}\left\|v_{2}\right\|^{2} \\
& \geq \frac{\varepsilon}{2}\left(\alpha\left\|y_{11}\right\|_{2}^{2}+\left\|v_{1}\right\|^{2}+\beta\left\|y_{21}\right\|_{1}^{2}+\left\|v_{2}\right\|^{2}\right) .
\end{aligned}
$$

Substituting (4.20) and (4.21) into (4.19) yields

$$
\begin{aligned}
& \frac{d}{d t}\left(\alpha\left\|y_{11}\right\|_{2}^{2}-p\left\|y_{11}\right\|_{1}^{2}+\left\|v_{1}\right\|^{2}+\beta\left\|y_{21}\right\|_{1}^{2}+\left\|v_{2}\right\|^{2}+\|u\|_{1}^{2}\left\|y_{11}\right\|_{1}^{2}\right) \\
& \quad+\varepsilon\left(\alpha\left\|y_{11}\right\|_{2}^{2}-2 p\left\|y_{11}\right\|_{1}^{2}+\left\|v_{1}\right\|^{2}+\beta\left\|y_{21}\right\|_{1}^{2}+\left\|v_{2}\right\|^{2}+2\|u\|_{1}^{2}\left\|y_{11}\right\|_{1}^{2}\right) \leq 0 .
\end{aligned}
$$

Let

$$
G(t)=\alpha\left\|y_{11}\right\|_{2}^{2}-p\left\|y_{11}\right\|_{1}^{2}+\left\|v_{1}\right\|^{2}+\beta\left\|y_{21}\right\|_{1}^{2}+\left\|v_{2}\right\|^{2}+\|u\|_{1}^{2}\left\|y_{11}\right\|_{1}^{2},
$$

then (3.1) ensures that

$$
G(t) \geq C(p)\left\|Y_{1}\right\|_{E}^{2}>0
$$

and

$$
\varepsilon\left(\alpha\left\|y_{11}\right\|_{2}^{2}-2 p\left\|y_{11}\right\|_{1}^{2}+\left\|v_{1}\right\|^{2}+\beta\left\|y_{21}\right\|_{1}^{2}+\left\|v_{2}\right\|^{2}+\|u\|_{1}^{2}\left\|y_{11}\right\|_{1}^{2}\right) \geq \frac{\varepsilon}{2} G(t) \quad \text { for } p \leq \frac{\alpha \sqrt{\lambda_{1}}}{3} .
$$


Thus, from (4.22) we conclude that

$$
\frac{d}{d t} G(t)+\frac{\varepsilon}{2} G(t) \leq 0
$$

By the Gronwall lemma, we get

$$
\begin{aligned}
G(0) & \leq e^{\frac{\varepsilon \tau}{2}} G(\tau) \\
& \leq e^{\frac{\varepsilon \tau}{2}}\left(\alpha\left\|u_{10}\right\|_{2}^{2}-p\left\|u_{10}\right\|_{1}^{2}+\left\|u_{11}+\varepsilon u_{10}\right\|^{2}+\beta\left\|u_{20}\right\|_{1}^{2}+\left\|u_{21}+\varepsilon u_{20}\right\|^{2}+\left\|u_{10}\right\|_{1}^{4}\right) .
\end{aligned}
$$

Noticing that

$$
\left\|Y_{1}(0)\right\|_{E}^{2}=\alpha\left\|y_{11}(0)\right\|_{2}^{2}+\left\|y_{11 t}(0)+\varepsilon y_{11}(0)\right\|^{2}+\beta\left\|y_{21}(0)\right\|_{1}^{2}+\left\|y_{21 t}(0)+\varepsilon y_{21}(0)\right\|^{2} \leq \frac{G(0)}{C(p)}
$$

we complete the proof.

Lemma 4.3 Assume that (1.4) holds, there exists a random radius $r_{2}(\omega)$ such that, for $P$ a.s. $\omega \in \Omega$,

$$
\left\|A^{\frac{1}{4}} Y_{2}\left(0, \omega ; Y_{2}(\tau, \omega)\right)\right\|_{E}^{2} \leq \frac{r_{1}^{2}(\omega)}{C(p)}
$$

where $Y_{2}=\left(\left(y_{12}, y_{12 t}+\varepsilon y_{12}-q_{B}(x) W_{1}(t)\right)^{T},\left(y_{22}, y_{22 t}+\varepsilon y_{22}-q_{S}(x) W_{2}(t)\right)^{T}\right)$ satisfies (4.16), provided that $p \leq \frac{\alpha \sqrt{\lambda_{1}}}{3}$.

Proof Provided that

$$
Y_{2}=\left(Y_{21}, Y_{22}\right)=\left(\left(y_{12}, y_{12 t}+\varepsilon y_{12}-q_{B}(x) W_{1}(t)\right)^{T},\left(y_{22}, y_{22 t}+\varepsilon y_{22}-q_{S}(x) W_{2}(t)\right)^{T}\right),
$$

then equation (4.16) can be reduced to

$$
\begin{cases}Y_{21 t}+L_{1} Y_{21}=H_{1}\left(Y_{21}, \omega\right), & Y_{21}(\tau)=\left(0,-q_{B}(x) W_{1}(\tau)\right)^{T}, \quad t \geq \tau, \\ Y_{22 t}+L_{2} Y_{22}=H_{2}\left(Y_{22}, \omega\right), & Y_{22}(\tau)=\left(0,-q_{S}(x) W_{2}(\tau)\right)^{T}, \quad t \geq \tau,\end{cases}
$$

where

$$
H_{1}\left(Y_{21}, \omega\right)=\left(\begin{array}{c}
q_{B}(x) W_{1}(t) \\
-k\left(u_{1}-u_{2}\right)^{+}-\left(p-\|u\|_{1}^{2}\right) A^{\frac{1}{2}} y_{12}-f_{B}\left(u_{1}\right)-\left(\delta_{1}-\varepsilon\right) q_{B}(x) W_{1}(t)
\end{array}\right)
$$

and

$$
H_{2}\left(Y_{22}, \omega\right)=\left(\begin{array}{c}
q_{S}(x) W_{2}(t) \\
k\left(u_{1}-u_{2}\right)^{+}-f_{S}\left(u_{2}\right)-\left(\delta_{2}-\varepsilon\right) q_{S}(x) W_{2}(t)
\end{array}\right) .
$$


Taking the inner product in $V_{2}$ of the first equation of (4.24) with $A^{\frac{1}{2}} Y_{21}$, and taking the inner product in $V_{1}$ of the second equation of (4.24) with $A^{\frac{1}{2}} Y_{22}$, we find that

$$
\begin{aligned}
& \frac{1}{2} \frac{d}{d t}\left\|A^{\frac{1}{4}} Y_{21}\right\|_{V_{2}}^{2}+\left(L_{1} Y_{21}, A^{\frac{1}{2}} Y_{21}\right)_{V_{2}}+\frac{1}{2} \frac{d}{d t}\left\|A^{\frac{1}{4}} Y_{22}\right\|_{V_{1}}^{2}+\left(L_{2} Y_{22}, A^{\frac{1}{2}} Y_{22}\right)_{V_{1}} \\
& \quad=\left(H_{1}\left(Y_{21}, \omega\right), A^{\frac{1}{2}} Y_{21}\right)_{V_{2}}+\left(H_{2}\left(Y_{22}, \omega\right), A^{\frac{1}{2}} Y_{22}\right)_{V_{1}},
\end{aligned}
$$

where

$$
\begin{aligned}
\left(H_{1}\left(Y_{21}, \omega\right), A^{\frac{1}{2}} Y_{21}\right)_{V_{2}}+\left(H_{2}\left(Y_{22}, \omega\right), A^{\frac{1}{2}} Y_{22}\right)_{V_{1}} \\
=\left(-k\left(u_{1}-u_{2}\right)^{+}-\left(p-\left\|u_{1}\right\|_{1}^{2}\right) A^{\frac{1}{2}} y_{12}-f_{B}\left(u_{1}\right)-\left(\delta_{1}-\varepsilon\right) q_{B}(x) W_{1}(t),\right. \\
\left.\quad A^{\frac{1}{2}}\left(y_{12 t}+\varepsilon y_{12}-q_{B}(x) W_{1}(t)\right)\right) \\
\quad+\left(k\left(u_{1}-u_{2}\right)^{+}-f_{S}\left(u_{2}\right)-\left(\delta_{2}-\varepsilon\right) q_{S}(x) W_{2}(t), A^{\frac{1}{2}}\left(y_{22 t}+\varepsilon y_{22}-q_{S}(x) W_{2}(t)\right)\right) \\
\quad+\left(A^{\frac{1}{2}} y_{12}, q_{B}(x) W_{1}(t)\right)_{2}+\left(A^{\frac{1}{2}} y_{22}, q_{S}(x) W_{2}(t)\right)_{1} .
\end{aligned}
$$

According to (4.3), we have

$$
\begin{aligned}
& \left(L_{1} Y_{21}, A^{\frac{1}{2}} Y_{21}\right)_{V_{2}}+\left(L_{2} Y_{22}, A^{\frac{1}{2}} Y_{22}\right)_{V_{1}} \\
& \geq \frac{1}{2} \frac{d}{d t}\left((\alpha-1)\left\|A^{\frac{1}{4}} y_{12}\right\|_{2}^{2}+(\beta-1)\left\|A^{\frac{1}{4}} y_{22}\right\|_{1}^{2}\right)+\frac{\varepsilon}{2}\left\|A^{\frac{1}{4}} Y_{2}\right\|_{E}^{2} \\
& \quad+\frac{\varepsilon}{4}\left(\alpha\left\|A^{\frac{1}{4}} y_{12}\right\|_{2}^{2}+\beta\left\|A^{\frac{1}{4}} y_{22}\right\|_{1}^{2}\right) \\
& \quad+\frac{\delta_{1}}{2}\left\|A^{\frac{1}{4}}\left(y_{12 t}+\varepsilon y_{12}-q_{B}(x) W_{1}(t)\right)\right\|^{2}+\frac{\delta_{2}}{2}\left\|A^{\frac{1}{4}}\left(y_{22 t}+\varepsilon y_{22}-q_{S}(x) W_{2}(t)\right)\right\|^{2} \\
& \quad+(1-\alpha)\left(A y_{12}, A^{\frac{1}{2}} q_{B}(x) W_{1}(t)\right)+(1-\beta)\left(A^{\frac{1}{2}} y_{22}, A^{\frac{1}{2}} q_{S}(x) W_{2}(t)\right) .
\end{aligned}
$$

Thanks to the Young inequality, we obtain

$$
\begin{aligned}
\alpha\left(A y_{12}, A^{\frac{1}{2}} q_{B}(x) W_{1}(t)\right)+\beta\left(A^{\frac{1}{2}} y_{22}, A^{\frac{1}{2}} q_{S}(x) W_{2}(t)\right) & \\
\leq & \frac{\varepsilon}{4}\left(\alpha\left\|A^{\frac{1}{4}} y_{12}\right\|_{2}^{2}+\beta\left\|A^{\frac{1}{4}} y_{22}\right\|_{1}^{2}\right)+\frac{\alpha}{\varepsilon}\left\|A^{\frac{1}{4}} q_{B}\right\|_{2}^{2}\left|W_{1}(t)\right|^{2} \\
& +\frac{\beta}{\varepsilon}\left\|A^{\frac{1}{4}} q_{S}\right\|_{1}^{2}\left|W_{2}(t)\right|^{2} .
\end{aligned}
$$

Below we estimate (4.26) one by one:

$$
\begin{aligned}
&-\left(\left(p-\left\|u_{1}\right\|_{1}^{2}\right) A^{\frac{1}{2}} y_{12}, A^{\frac{1}{2}}\left(y_{12 t}+\varepsilon y_{12}-q_{B}(x) W_{1}(t)\right)\right) \\
& \geq \frac{1}{2} \frac{d}{d t}\left(\left\|u_{1}\right\|_{1}^{2}\left\|y_{12}\right\|_{2}^{2}-p\left\|y_{12}\right\|_{2}^{2}\right)+\frac{\varepsilon}{2}\left(\left\|u_{1}\right\|_{1}^{2}\left\|y_{12}\right\|_{2}^{2}-p\left\|y_{12}\right\|_{2}^{2}\right) \\
& \quad-\left(\frac{\varepsilon}{2}|p|+\frac{1}{2}\left(|p|+\left\|u_{1}\right\|_{1}^{2}\right)^{2}\right)\left\|y_{12}\right\|_{2}^{2}-\frac{1}{2}\left\|A^{\frac{1}{2}} q_{B}\right\|^{2}\left|W_{1}(t)\right|^{2}-\left\|u_{1}\right\|_{2}^{3}\left\|u_{1 t}\right\| ; \\
& \mid-\left(k\left(u_{1}-u_{2}\right)^{+}, A^{\frac{1}{2}}\left(y_{12 t}+\varepsilon y_{12}-q_{B}(x) W_{1}(t)\right)\right)+\left(k\left(u_{1}-u_{2}\right)^{+},\right. \\
&\left.\quad A^{\frac{1}{2}}\left(y_{22 t}+\varepsilon y_{22}-q_{S}(x) W_{2}(t)\right)\right) \mid
\end{aligned}
$$




$$
\begin{aligned}
& \leq k\left\|A^{\frac{1}{4}} u_{1}\right\|\left\|A^{\frac{1}{4}}\left(y_{12 t}+\varepsilon y_{12}-q_{B}(x) W_{1}(t)\right)\right\| \\
&+k\left\|A^{\frac{1}{4}} u_{2}\right\|\left\|A^{\frac{1}{4}}\left(y_{12 t}+\varepsilon y_{12}-q_{S}(x) W_{1}(t)\right)\right\| \\
&+k\left\|A^{\frac{1}{4}} u_{1}\right\|\left\|A^{\frac{1}{4}}\left(y_{22 t}+\varepsilon y_{22}-q_{S}(x) W_{2}(t)\right)\right\| \\
&+k\left\|A^{\frac{1}{4}} u_{2}\right\|\left\|A^{\frac{1}{4}}\left(y_{22 t}+\varepsilon y_{22}-q_{S}(x) W_{2}(t)\right)\right\| \\
& \leq\left(\frac{4 k^{2}}{\delta_{1}}+\frac{4 k^{2}}{\delta_{2}}\right)\left\|A^{\frac{1}{4}} u_{1}\right\|^{2}+\frac{\delta_{1}}{8}\left\|A^{\frac{1}{4}}\left(y_{12 t}+\varepsilon y_{12}-q_{B}(x) W_{1}(t)\right)\right\|^{2} \\
&+\left(\frac{4 k^{2}}{\delta_{1}}+\frac{4 k^{2}}{\delta_{2}}\right)\left\|A^{\frac{1}{4}} u_{2}\right\|^{2}+\frac{\delta_{2}}{8}\left\|A^{\frac{1}{4}}\left(y_{22 t}+\varepsilon y_{22}-q_{S}(x) W_{2}(t)\right)\right\|^{2} ; \\
&\left(\left(\delta_{1}-\varepsilon\right) q_{B}(x) W_{1}(t), A^{\frac{1}{2}}\left(y_{12 t}+\varepsilon y_{12}-q_{B}(x) W_{1}(t)\right)\right) \\
& \quad+\left(\left(\delta_{2}-\varepsilon\right) q_{S}(x) W_{2}(t), A^{\frac{1}{2}}\left(y_{22 t}+\varepsilon y_{22}-q_{S}(x) W_{2}(t)\right)\right) \\
& \leq 2 \delta_{1}\left\|A^{\frac{1}{4}} q_{B}\right\|^{2}\left|W_{1}(t)\right|^{2}+\frac{\delta_{1}}{8}\left\|A^{\frac{1}{4}}\left(y_{12 t}+\varepsilon y_{12}-q_{B}(x) W_{1}(t)\right)\right\|^{2} \\
&+2 \delta_{2}\left\|A^{\frac{1}{4}} q_{S}\right\|^{2}\left|W_{2}(t)\right|^{2}+\frac{\delta_{2}}{8}\left\|A^{\frac{1}{4}}\left(y_{22 t}+\varepsilon y_{22}-q_{S}(x) W_{2}(t)\right)\right\|^{2} .
\end{aligned}
$$

Besides, by (1.4), (4.1), and the Sobolev embedding theorem, we show that $f_{B}^{\prime}(s), f_{S}^{\prime}(s)$ are uniformly bounded in $L^{\infty}$, that is,

$$
\left|\frac{d}{d s} f_{B}^{\prime}(s)\right|_{L^{\infty}} \leq C_{0}\left(|u|_{L^{\infty}}^{\gamma-1}+1\right) \leq C_{3}\left(\|u\|_{2}^{\gamma-1}+1\right) \leq C_{4} R_{1}^{\gamma-1}(t, \omega)
$$

similarly,

$$
\left|\frac{d}{d s} f_{S}^{\prime}(s)\right|_{L^{\infty}} \leq C_{4} R_{1}^{\gamma-1}(t, \omega)
$$

Combining with (4.32) and (4.33), it follows that

$$
\begin{aligned}
\left|-\left(f_{B}\left(u_{1}\right), A^{\frac{1}{2}}\left(y_{12 t}+\varepsilon y_{12}-q_{B}(x) W_{1}(t)\right)\right)-\left(f_{S}\left(u_{2}\right), A^{\frac{1}{2}}\left(y_{22 t}+\varepsilon y_{22}-q_{S}(x) W_{2}(t)\right)\right)\right| \\
\leq\left\|A^{\frac{1}{4}} f_{B}\left(u_{1}\right)\right\|\left\|A^{\frac{1}{4}}\left(y_{12 t}+\varepsilon y_{12}-q_{B}(x) W_{1}(t)\right)\right\| \\
\quad+\left\|A^{\frac{1}{4}} f_{S}\left(u_{2}\right)\right\|\left\|A^{\frac{1}{4}}\left(y_{22 t}+\varepsilon y_{22}-q_{S}(x) W_{2}(t)\right)\right\| \\
\leq \leq \frac{2 C_{5} R_{1}^{2(\gamma-1)}(t, \omega)}{\sqrt{\lambda_{1}} \delta_{1}}\left\|u_{1}\right\|_{2}^{2}+\frac{\delta_{1}}{8}\left\|A^{\frac{1}{4}}\left(y_{12 t}+\varepsilon y_{12}-q_{B}(x) W_{1}(t)\right)\right\|^{2} \\
\quad+\frac{C_{5} R_{1}^{2(\gamma-1)}(t, \omega)}{\delta_{2}}\left\|u_{2}\right\|_{1}^{2}+\frac{\delta_{2}}{4}\left\|A^{\frac{1}{4}}\left(y_{22 t}+\varepsilon y_{22}-q_{S}(x) W_{2}(t)\right)\right\|^{2}
\end{aligned}
$$

Thus, collecting all (4.26)-(4.34) from (4.25) yields, for $\tau \leq T(B)$,

$$
\begin{aligned}
\frac{d}{d t}\left(\left\|A^{\frac{1}{4}} Y_{2}\right\|_{E}^{2}-p\left\|y_{12}\right\|_{2}^{2}+\left\|u_{1}\right\|_{1}^{2}\left\|y_{12}\right\|_{2}^{2}\right)+\varepsilon\left(\left\|A^{\frac{1}{4}} Y_{2}\right\|_{E}^{2}-p\left\|y_{12}\right\|_{2}^{2}+\left\|u_{1}\right\|_{1}^{2}\left\|y_{12}\right\|_{2}^{2}\right) \\
\leq\left(\frac{8 k^{2}+4 C_{5} R_{1}^{2(\gamma-1)}(t, \omega)}{\sqrt{\lambda_{1}} \delta_{1}}+\frac{8 k^{2}}{\sqrt{\lambda_{1}} \delta_{2}}+\varepsilon|p|+\left(|p|+\left\|u_{1}\right\|_{1}^{2}\right)^{2}\right)\left\|u_{1}\right\|_{2}^{2} \\
\quad+\left(\frac{8 k^{2}+2 C_{5} R_{1}^{2(\gamma-1)}(t, \omega)}{\delta_{2}}+\frac{8 k^{2}}{\delta_{1}}\right)\left\|u_{2}\right\|_{1}^{2}+2\left\|u_{1}\right\|_{2}^{3}\left\|u_{1 t}\right\|
\end{aligned}
$$




$$
\begin{aligned}
& +\left(\frac{2 \alpha}{\varepsilon}\left\|A^{\frac{1}{4}} q_{B}\right\|_{2}^{2}+4 \delta_{1}\left\|A^{\frac{1}{4}} q_{B}\right\|^{2}+\left\|A^{\frac{1}{2}} q_{B}\right\|^{2}\right)\left|W_{1}(t)\right|^{2} \\
& +\left(\frac{2 \beta}{\varepsilon}\left\|A^{\frac{1}{4}} q_{S}\right\|_{1}^{2}+4 \delta_{2}\left\|A^{\frac{1}{4}} q_{S}\right\|^{2}\right)\left|W_{2}(t)\right|^{2} .
\end{aligned}
$$

We have

$$
\begin{aligned}
& \frac{d}{d t} H(t)+\varepsilon H(t) \\
& \quad \leq C_{6} R_{1}^{2 \gamma}(t, \omega)+C_{7} R_{1}^{6}(t, \omega)+\left(\frac{2 \alpha}{\varepsilon}\left\|A^{\frac{1}{4}} q_{B}\right\|_{2}^{2}+4 \delta_{1}\left\|A^{\frac{1}{4}} q_{B}\right\|^{2}+\left\|A^{\frac{1}{2}} q_{B}\right\|^{2}\right)\left|W_{1}(t)\right|^{2} \\
& \quad+\left(\frac{2 \beta}{\varepsilon}\left\|A^{\frac{1}{4}} q_{S}\right\|_{1}^{2}+4 \delta_{2}\left\|A^{\frac{1}{4}} q_{S}\right\|^{2}\right)\left|W_{2}(t)\right|^{2}, \quad \tau \leq t \leq 0,
\end{aligned}
$$

where

$$
H(t)=\left\|A^{\frac{1}{4}} Y_{2}\right\|_{E}^{2}-p\left\|y_{12}\right\|_{2}^{2}+\left\|u_{1}\right\|_{1}^{2}\left\|y_{12}\right\|_{2}^{2} .
$$

Noting (3.1) and $C(p)$ defined as in Lemma 4.2, it follows that $H(t) \geq C(p)\left\|A^{\frac{1}{4}} Y_{2}\right\|_{E}^{2}>0$. Applying the Gronwall lemma, we arrive at

$$
\begin{aligned}
H(0) \leq & e^{\varepsilon \tau}\left(\left\|A^{\frac{1}{4}} q_{B}\right\|^{2}\left|W_{1}(\tau)\right|^{2}+\left\|A^{\frac{1}{4}} q_{S}\right\|^{2}\left|W_{2}(\tau)\right|^{2}\right) \\
& +\int_{\tau}^{0} e^{\varepsilon s}\left(C_{6} R_{1}^{2 \gamma}(t, \omega)+C_{7} R_{1}^{6}(t, \omega)\right) d s \\
& +\left(\frac{2 \alpha}{\varepsilon}\left\|A^{\frac{1}{4}} q_{B}\right\|_{2}^{2}+4 \delta_{1}\left\|A^{\frac{1}{4}} q_{B}\right\|^{2}+\left\|A^{\frac{1}{2}} q_{B}\right\|^{2}\right) \int_{\tau}^{0} e^{\varepsilon s}\left|W_{1}(s)\right|^{2} d s \\
& +\left(\frac{2 \beta}{\varepsilon}\left\|A^{\frac{1}{4}} q_{S}\right\|_{1}^{2}+4 \delta_{2}\left\|A^{\frac{1}{4}} q_{S}\right\|^{2}\right) \int_{\tau}^{0} e^{\varepsilon s}\left|W_{2}(s)\right|^{2} d s .
\end{aligned}
$$

It is easy to see that

$$
A^{\frac{1}{4}} Y_{2}\left(0, \omega ; Y_{2}(\tau, \omega)\right) \|_{E}^{2} \leq \frac{H(0)}{C(p)} .
$$

Set

$$
\begin{aligned}
r_{1}^{2}(\omega)= & \left\|A^{\frac{1}{4}} q_{B}\right\|^{2} \sup _{\tau \leq 0} e^{\varepsilon \tau}\left|W_{1}(\tau)\right|^{2}+\left\|A^{\frac{1}{4}} q_{S}\right\|^{2} \sup _{\tau \leq 0} e^{\varepsilon \tau}\left|W_{2}(\tau)\right|^{2} \\
& +\int_{-\infty}^{0} e^{\varepsilon s}\left(C_{6} R_{1}^{2 \gamma}(t, \omega)+C_{7} R_{1}^{6}(t, \omega)\right) d s \\
& +\left(\frac{2 \alpha}{\varepsilon}\left\|A^{\frac{1}{4}} q_{B}\right\|_{2}^{2}+4 \delta_{1}\left\|A^{\frac{1}{4}} q_{B}\right\|^{2}+\left\|A^{\frac{1}{2}} q_{B}\right\|^{2}\right) \int_{-\infty}^{0} e^{\varepsilon s}\left|W_{1}(s)\right|^{2} d s \\
& +\left(\frac{2 \beta}{\varepsilon}\left\|A^{\frac{1}{4}} q_{S}\right\|_{1}^{2}+4 \delta_{2}\left\|A^{\frac{1}{4}} q_{S}\right\|^{2}\right) \int_{-\infty}^{0} e^{\varepsilon s}\left|W_{2}(s)\right|^{2} d s .
\end{aligned}
$$

Since $\lim _{t \rightarrow \infty} \frac{W_{1}(t)}{t}=0, \lim _{t \rightarrow \infty} \frac{W_{2}(t)}{t}=0, r_{1}^{2}(\omega)$ is finite $P$-a.s. By (4.35)-(4.37), we have

$$
\left\|A^{\frac{1}{4}} Y_{2}\left(0, \omega ; Y_{2}(\tau, \omega)\right)\right\|_{E}^{2} \leq \frac{r_{1}^{2}(\omega)}{C(p)}
$$

for all $\left(\left(u_{10}, u_{11}+\varepsilon u_{10}\right)^{T},\left(u_{20}, u_{21}+\varepsilon u_{20}\right)^{T}\right) \in B$, and all $\tau \leq T(B)$. 
Theorem 4.4 Let (1.4)-(1.6) hold, $p \leq \frac{\alpha \sqrt{\lambda_{1}}}{3}, q_{B}(x) \in H^{3}(U) \cap H_{0}^{2}(U)$, and $q_{S}(x) \in H^{2}(U) \cap$ $H_{0}^{1}(U)$. Then the random dynamical system $S(t, \omega)$ possesses a nonempty compact random attractor $\mathcal{A}$.

Proof Let $B_{1}(\omega)$ be the ball of $H^{3}(U) \times H_{0}^{1}(U) \times\left(H^{2}(U) \cap H_{0}^{1}(U)\right) \times H_{0}^{1}(U)$ of radius $\frac{r_{1}(\omega)}{\sqrt{C(p)}}$. From the compact embedding $H^{3}(U) \times H_{0}^{1}(U) \times\left(H^{2}(U) \cap H_{0}^{1}(U)\right) \times H_{0}^{1}(U)$ into $E$, it follows that $B_{1}(\omega)$ is compact in $E$ for every bounded non-random set $B$ of $E$ and any $\varphi(0) \in \bar{S}_{\varepsilon}\left(t, \theta_{-t} \omega\right) B$. From Lemma 4.2, we know that $Y_{2}(0)=\varphi(0)-Y_{1}(0) \in B_{1}(\omega)$, where $Y_{2}(t, \omega)$ is given by (4.24). Therefore, by means of Lemma 4.3 , for $\tau \leq 0$,

$$
\begin{aligned}
& \inf _{l(0) \in B_{1}(\omega)}\|\varphi(0)-l(0)\|_{E}^{2} \\
& \leq\left\|Y_{1}(0)\right\|_{E}^{2} \\
& \leq \frac{e^{\frac{\varepsilon \tau}{2}}}{C(p)}\left(\alpha\left\|u_{10}\right\|_{2}^{2}-p\left\|u_{10}\right\|_{1}^{2}+\left\|u_{11}+\varepsilon u_{10}\right\|^{2}+\beta\left\|u_{20}\right\|_{1}^{2}+\left\|u_{21}+\varepsilon u_{20}\right\|^{2}\right) .
\end{aligned}
$$

Thus, for all $t \geq 0$,

$$
\begin{aligned}
& \mathrm{d}\left(\bar{S}_{\varepsilon}\left(t, \theta_{-t} \omega\right) B, B_{1}(\omega)\right) \\
& \quad \leq \frac{e^{-\frac{\varepsilon t}{2}}}{C(p)}\left(\alpha\left\|u_{10}\right\|_{2}^{2}-p\left\|u_{10}\right\|_{1}^{2}+\left\|u_{11}+\varepsilon u_{10}\right\|^{2}+\beta\left\|u_{20}\right\|_{1}^{2}+\left\|u_{21}+\varepsilon u_{20}\right\|^{2}\right) .
\end{aligned}
$$

Finally, from relation (3.9) between $S(t, \omega)$ and $\bar{S}_{\varepsilon}(t, \omega)$, one can easily obtain that, for any non-random bounded $B \subset E$ P-a.s.,

$$
\mathrm{d}\left(S\left(t, \theta_{-t} \omega\right) B, B_{1}(\omega)\right) \rightarrow 0 \quad \text { as } t \rightarrow+\infty .
$$

Hence, the RDS $S(t, \omega)$ associated with (3.7) possesses a uniformly attracting compact set $B_{1}(\omega) \subset E$. Then, applying Theorem 2.5 , we complete the proof.

\section{Acknowledgements}

The authors would like to thank the reviewers and the editors for their valuable suggestions and comments.

Funding

This work was supported by the NSF of China (11771449, 11561064), the NSF of Gansu Province (17JR5RA069), the University Project of Gansu Province (2017B-90), the Project of Northwest Normal University (NWNU-LKQN-16-16, NWNU-LKQN-18-14), and China Postdoctoral Science Foundation (2017M623380).

\section{Availability of data and materials}

Not applicable.

\section{Competing interests}

The authors declare that they have no competing interests.

\section{Authors' contributions}

All authors contributed equally to the writing of this paper. All authors read and approved the final manuscript.

\section{Author details}

${ }^{1}$ College of Liberal Arts and Sciences, National University of Defense Technology, Changsha, P.R. China. ${ }^{2}$ College of Mathematics and Statistics, Northwest Normal University, Lanzhou, P.R. China.

\section{Publisher's Note}

Springer Nature remains neutral with regard to jurisdictional claims in published maps and institutional affiliations. 


\section{References}

1. Lazer, A.C., McKenna, P.J.: Large-amplitude periodic oscillations in suspension bridges: some new connection with nonlinear analysis. SIAM Rev. 32(4), 537-578 (1990)

2. Ahmed, N.U., Harbi, H.: Mathematical analysis of dynamical models of suspension bridges. SIAM J. Appl. Math. 58(3), 853-874 (1998)

3. Humphreys, L.D.: Numerical mountain pass solutions of a suspension bridge equation. Nonlinear Anal. 28(11) 1811-1826 (1997)

4. McKenna, P.J., Walter, W.: Nonlinear oscillation in a suspension bridges. Arch. Ration. Mech. Anal. 98, 167-177 (1987) Results: Nonlinear Anal. 39, 731-743 (2000)

5. Zhong, C.K., Ma, Q.Z., Sun, C.Y.: Existence of strong solutions and global attractors for the suspension bridge equations. Nonlinear Anal. 67, 442-454 (2007)

6. Park, J.Y., Kang, J.R.: Global attractors for the suspension bridge equations with nonlinear damping. Q. Appl. Math. 69, 465-475 (2011)

7. Park, J.Y., Kang, J.R.: Pullback $\mathcal{D}$-attractors for non-autonomous suspension bridge equations. Nonlinear Anal. 71, 4618-4623 (2009)

8. Park, J.Y., Kang, J.R.: Uniform attractor for non-autonomous suspension bridge equations with localized damping. Math. Methods Appl. Sci. 34, 487-496 (2011)

9. Litcanu, G.: A mathematical model of suspension bridges. Appl. Math. 49(1), 39-55 (2004)

10. Malik, J.: Mathematical modelling of cable-stayed bridge: existence, uniqueness, continuous dependence on data, homogenization of cable systems. Appl. Math. 49(1), 1-38 (2004)

11. Holubová, G., Matas, A.: Initial-boundary value problem for the nonlinear string-beam system. J. Math. Anal. Appl. 288, 784-802 (2003)

12. Ma, Q.Z., Zhong, C.K.: Existence of global attractors for the coupled suspension bridge equations. J. Math. Anal. Appl. $308,365-379(2005)$

13. Ma, Q.Z., Zhong, C.K.: Existence of strong solutions and global attractors for the coupled suspension bridge equations. J. Differ. Equ. 246, 3755-3775 (2009)

14. Ma, Q.Z., Wang, S.P., Chen, X.B.: Uniform attractors for the coupled suspension bridge equations. Appl. Math. Comput. 217, 6604-6615 (2011)

15. Ma, Q.Z., Wang, B.L.: Existence of pullback attractors for the coupled suspension bridge equations. Electron. J. Differ. Equ. 2011, 16 (2011)

16. Kang, J.R.: Pullback attractors for the non-autonomous coupled suspension bridge equations. Appl. Math. Comput. 219, 8747-8758 (2013)

17. Woinowsky-Krieger, S.: The effect of an axial force on the vibration of hinged bars. J. Appl. Mech. 17, 35-36 (1950)

18. Bochicchio, I., Giorgi, C., Vuk, E.: Long-term damped dynamics of the extensible suspension bridge. Int. J. Differ. Equ. $2010,383420(2010)$

19. Bochicchio, I., Giorgi, C., Vuk, E.: Asymptotic dynamical of nonlinear coupled suspension bridge equations. J. Math Anal. Appl. 402, 319-333 (2013)

20. Ahmed, N.U.: A general mathematical framework for stochastic analysis of suspension bridges. Nonlinear Anal., Real World Appl. 1, 457-483 (2000)

21. Ma, Q.Z., Xu, L.: Random attractors for the extensible suspension bridge equation with white noise. Comput. Math. Appl. 70, 2895-2903 (2015)

22. Ma, Q.Z., Xu, L.: Random attractors for the coupled suspension bridge equations with white noises. Appl. Math. Comput. 306, 38-48 (2017)

23. Crauel, H. Flandoli, F.: Attractors for random dynamical systems. Probab. Theory Relat. Fields 100, 365-393 (1994)

24. Crauel, H., Debussche, A., Flandoli, F.: Random attractors. J. Dyn. Differ. Equ. 9, 307-314 (1997)

25. Arnold, L.: Random Dynamical Systems. Springer, New York (1998)

26. Fan, X.M.: Random attractor for a damped sine-Gordon equation with white noise. Pac. J. Math. 216, 63-76 (2004)

27. Fan, X.M., Wang, Y.G.: Fractal dimensional of attractors for a stochastic wave equation with nonlinear damping and white noise. Stoch. Anal. Appl. 25, 381-396 (2007)

28. Yang, M.H., Kloeden, P.E.: Random attractors for stochastic semi-linear degenerate parabolic equation. Nonlinear Anal., Real World Appl. 12, 2811-2821 (2011)

29. Yang, M.H., Duan, J.Q., Kloeden, P.E.: Asymptotic behavior of solutions for random wave equation with nonlinear damping and white noise. Nonlinear Anal., Real World Appl. 12,464-478 (2011)

30. Ma, W.J., Ma, Q.Z.: Attractors for stochastic strongly damped plate equations with additive noise. Electron. J. Differ. Equ. 2013, $111(2013)$

31. Pazy, A.: Semigroup of Linear Operators and Applications to Partial Differential Equations. Appl. Math. Sci. Berlin. Springer, New York (1983) 